\title{
Cationic Liposome- Multi-Walled Carbon Nanotubes Hybrids for Dual siPLKI and Doxorubicin Delivery In Vitro
}

\author{
Sara Pereira',3 • Jin Lee' • Noelia Rubio' - Hatem A. F. M. Hassan' • Izzat Bin Mohamed Suffian' • Julie T. W. Wang' •
} Rebecca Klippstein ' Belén Ballesteros ${ }^{2} \cdot$ Wafa' T. Al-Jamal $^{3} \cdot$ Khuloud T. Al-Jamal $^{1}$

Received: 23 December 2014 / Accepted: 5 May 2015 / Published online: 18 June 2015

(C) The Author(s) 2015. This article is published with open access at Springerlink.com

\begin{abstract}
Purpose To formulate $f$-MWNTs-cationic liposome hybrids for the simultaneous delivery of siPLK1 and doxorubicin to cancer cells.

Method f-MWNTs-cationic liposome hybrids were prepared by the thin film hydration method where the lipid film was hydrated with $100 \mu \mathrm{g} / \mathrm{ml}$ or $1 \mathrm{mg} / \mathrm{ml}$ of ox-MWNTs-NH ${ }_{3}^{+}$ or MWNTs- $\mathrm{NH}_{3}{ }^{+}$in $5 \%$ dextrose. siRNA complexation and protection ability was determined by agarose gel electrophoresis. $f$-MWNTs and liposome interaction was evaluated using Nile Red (NR) fluorescence spectroscopy. Cellular uptake in A549 cells was assessed by flow cytometry. Silencing of target proteins was determined by Luciferase and MTT assays. Sub-G1 analysis was performed to evaluate apoptosis following co-delivery of siPLK1 and Doxorubicin (Dox).

Results Zeta potential and siRNA complexation profile obtained for all hybrids were comparable to those achieved with cationic liposomes. ox-MWNTs- $\mathrm{NH}_{3}{ }^{+}$showed greater extent of interaction with cationic liposomes compared to MWNTs-
\end{abstract}

Electronic supplementary material The online version of this article (doi: | 0. I007/s | 1095-0 | 5- | 707- I) contains supplementary material, which is available to authorized users.

Wafa' T. Al-Jamal

wafa.al-jamal@uea.ac.uk

Khuloud T. Al-Jamal

khuloud.al-jamal@kcl.ac.uk

Institute of Pharmaceutical Science, King's College

London, Franklin-Wilkins Building, I 50 Stamford Street, London SE I

$9 \mathrm{NH}, \mathrm{UK}$

2 ICN2 - Institut de Catala de Nanociencia i Nanotecnologia, Campus UAB, 08193 Bellaterra, Barcelona, Spain

3 School of Pharmacy, University of East Anglia, Norwich Research Park, Norwich NR4 7TJ, UK

$\mathrm{NH}_{3}{ }^{+}$. ox-MWNTs- $\mathrm{NH}_{3}{ }^{+}$was able to protect siRNA from nuclease-mediated degradation. Enhanced cellular uptake of both the carrier and loaded siRNA in A549 cell, were observed for this hybrid compared to the liposomal carrier. A synergistic pro-apoptotic effect was obtained when siPLK1 silencing was combined with doxorubicin treatment for the hybrid:siRNA complexes compared to the lipoplexes, in A549 cells in vitro.

Conclusions f-MWNTs-cationic liposome hybrid designed in this study can serve as a potential vehicle for the co-delivery of siRNA and cytotoxic drugs to cancer cells in vitro.

KEY WORDS A549 . cancer cells · gene silencing . multi-walled carbon nanotubes · siRNA

\section{ABBREVIATIONS}

$\mathrm{I}-\mathrm{H}$

I-L

2-L

chol

CNTS

DLSI

DOTAP

Dox

f-CNTs
ox-MWNTs- $\mathrm{NH}_{3}{ }^{+}$-liposome hybrid (f-MWNT:total lipid mass ratio $35.8 \%: 64.2 \%$ ) ox-MWNTs- $\mathrm{NH}_{3}{ }^{+}$-liposome hybrid (f-MWNT:total lipid mass ratio 5.3\%:94.7\%)

MWNTs- $\mathrm{NH}_{3}{ }^{+}$-liposome hybrid (f-MWNT:total lipid mass ratio $35.8 \%: 64.2 \%)$

MWNTs- $\mathrm{NH}_{3}{ }^{+}$-liposome hybrid (f-MWNT:total lipid mass ratio 5.3\%:94.7\%)

Cholesterol

Carbon nanotubes

Dynamic light scattering

1,2-dioleoyl-3-trimethylammoniumpropane

Doxorubicin

Functionalized carbon nanotubes 


\begin{tabular}{|c|c|}
\hline$f-M W N T s$ & $\begin{array}{l}\text { Functionalized multi-walled carbon } \\
\text { nanotubes }\end{array}$ \\
\hline L & Liposome \\
\hline Luc & Luciferase \\
\hline $\mathrm{MFI}$ & Mean Fluorescence Intensity \\
\hline MTT & $\begin{array}{l}\text { 3-(4,5-dimethylthiazol-2-yl)-2,5-diphe- } \\
\text { nyltetrazolium bromide }\end{array}$ \\
\hline 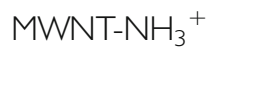 & $\begin{array}{l}\text { Ammonium-functionalized multi- } \\
\text { walled carbon nanotubes }\end{array}$ \\
\hline NR & Nile Red \\
\hline $\mathrm{ox}-\mathrm{MWNT}-\mathrm{NH}_{3}{ }^{+}$ & $\begin{array}{l}\text { Ammonium-functionalized oxidized } \\
\text { multi-walled carbon nanotubes }\end{array}$ \\
\hline PDI & Polydispersity index \\
\hline PLKI & Polo-like kinase 1 \\
\hline RISC & RNA-induced silencing complex \\
\hline RNAse & Ribonuclease \\
\hline siNEG & non-coding siRNA \\
\hline siRNA & Small interfering RNA \\
\hline SSC & Sideward scattering \\
\hline
\end{tabular}

\section{INTRODUCTION}

Small interfering RNA (siRNA) has emerged as a therapeutic strategy for various diseases, including cancer, due to its target-specific gene silencing. Moreover, down-regulation of genes that are overexpressed in tumors by siRNA presents advantages over plasmid DNA therapy. As siRNA acts at the cytoplasm level, it does not need to cross the nuclear envelope contrary to DNA which needs to be delivered to the cell nucleus (1). However, due to its high molecular weight and negative charge, cellular uptake and subsequent intracellular trafficking to the effector complex (RNA-induced silencing complex, RISC) are hampered and represent major technical hurdles for the efficacy of siRNA (2). Thus, in the past few years researchers have focused on the development of suitable delivery systems that would make clinical practice of siRNA therapy possible. Approaches to overcome these limitations have relied on non-viral siRNA carriers based on cationic liposomes or polymers able to spontaneously complex and condense genetic materials (3). Nevertheless, these systems are characterized by a lack of efficiency accompanied by a high level of toxicity rendering them mostly inadequate for in vivo applications (2).

Recently, a unique type of nonviral gene vector has emerged, based on functionalized carbon nanotubes $(f$-CNTs $)(4)$. CNTs are ordered structures with high aspect ratio and unique features such as high mechanical strength, high thermal and electrical conductivity (5). In addition, during the last decade, their potential use in the biomedical field has also attracted great interest due to CNTs ability to translocate into the cytoplasm by directly crossing the plasma membrane via an endocytosis-independent route where they act like "nanoneedles" without inducing apoptosis (4-6). The main problem associated with the use of GNTs is their hydrophobicity which leads to poor dispersibility. However, this can be circumvented by surface chemical functionalization, improving CNTs dispersibility in physiological media and consequently broadening the spectrum of their potential biological applications (7-9).

In the present study, we aimed at formulating functionalized multi-walled carbon nanotubes ( $f$-MWNT)-cationic liposome hybrids for the co-delivery of siRNA and a cytotoxic drug in vitro. Doxorubicin (Dox) was chosen as the model drug as it is commonly used in the treatment of a wide range of cancers. Polo-like kinase 1 (PLK1) is a serine/threonine kinase with key roles in cell division and checkpoint regulation of mitosis. PLK1 is overexpressed in several types of cancer and correlates with high tumor grades. siRNA-mediated silencing of PLK 1 in several cancer cell lines was shown to result in decreased cell viability with induction of apoptosis, defects in several mitosis processes and G2/M phase arrest (10). Other studies showed that PLK1 down-regulation sensitizes cancer cells to chemotherapy and can revert chemoresistance (11-14).

In this study, a series of $f$-MWNT-cationic liposome hybrids were formulated and tested for their siRNA binding ability, stability, cellular uptake, in vitro gene silencing efficiency, induction of apoptosis and siPLK1 and Dox co-delivery efficiency. Liposomes consisted of 1,2-dioleoyl-3-trimethylammonium-propane (DOTAP) and cholesterol (DOTAP:chol, 2:1 molar ratio), one of the most widely used transfection agents. Two types of $f$-MWNTs were used: $\mathrm{MWNT}_{\mathrm{NHH}}{ }^{+}(-455.5+/-$ $185.42 \mathrm{~nm}$ in length, 20-30 $\mathrm{nm}$ in diameter) and oxMWNT- $\mathrm{NH}_{3}{ }^{+}(-277.5+/-152.93 \mathrm{~nm} \mathrm{~nm}$ in length, 20-30 nm in diameter). $f$-MWNT-liposome hybrids were prepared at two different $f$-MWNT:total lipid mass ratios $(5.3 \%: 94.7 \%$ and $35.8 \%: 64.2 \%)$.

\section{MATERIALS AND METHODS}

\section{Materials}

Pristine MWNTs were purchased from Nanostructured and Amorphous Materials Inc. (Houston, USA); Batch: 1237YJS $95 \%$, outer diameters between 20 and $30 \mathrm{~nm}$, and lengths between 0.5 and $2 \mu \mathrm{m}$ ). ox-MWNT- $\mathrm{NH}_{3}{ }^{+}$(type I) and MWNT- $\mathrm{NH}_{3}{ }^{+}$(type 2 ) (Fig. 1) were functionalized using 1, 3-dipolar cycloaddition as described by Prato and co-workers (15). 1,2-dioleoyl-3-trimethylammonium-propane (chloride salt) (DOTAP, 99\%) was obtained from Avanti Polar Lipid (USA). Cholesterol, chloroform, methanol, dextrose, doxorubicin hydrochloride (Dox), MTT (3-(4,5-dimethylthiazol-2yl)-2,5-diphenyltetrazolium bromide), dimethylsulfoxide 


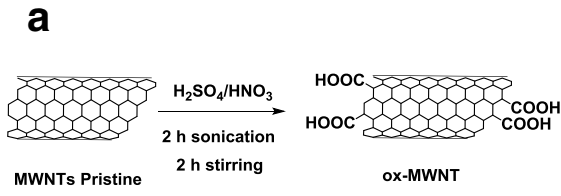

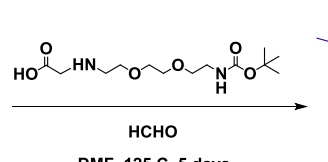
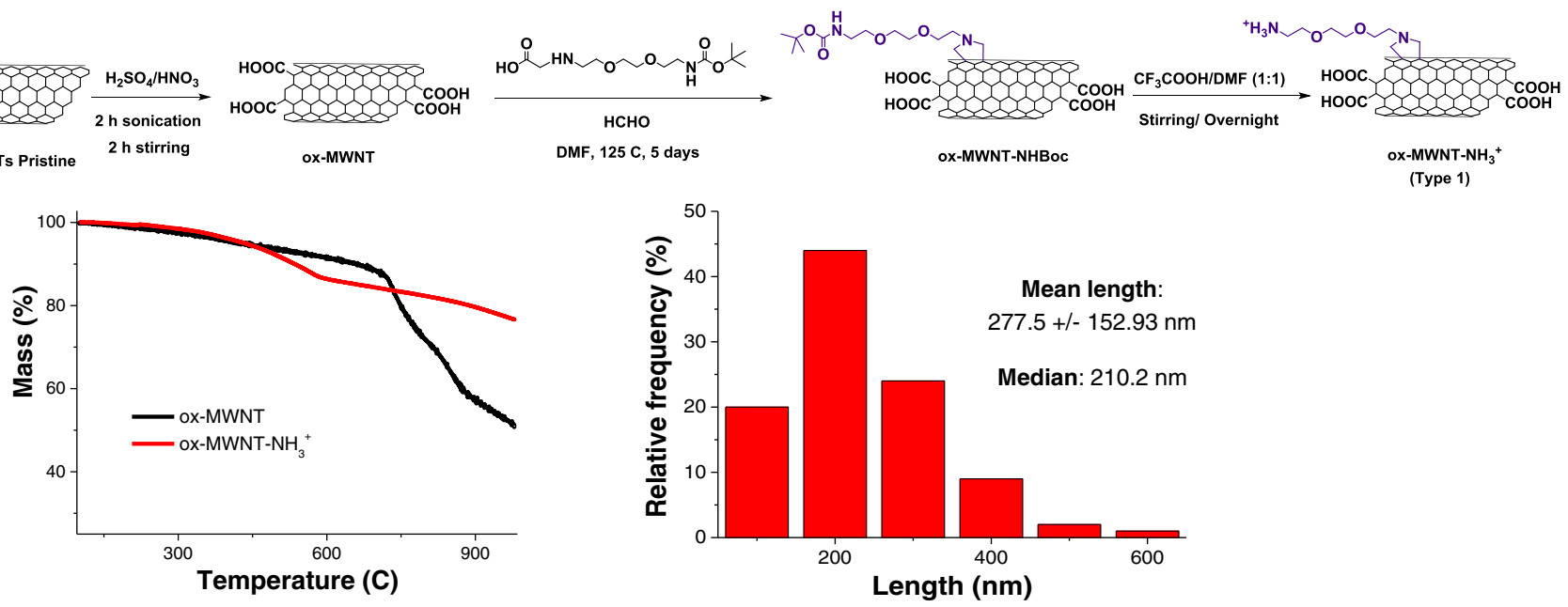

b
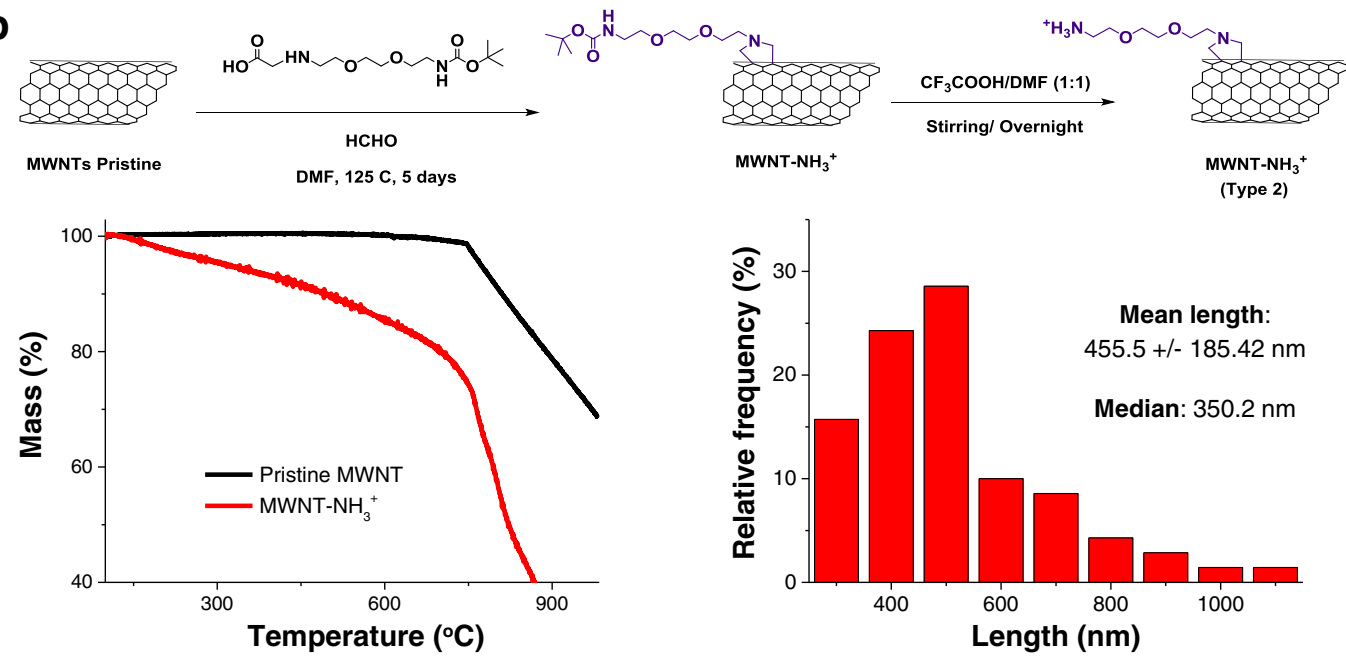

Fig. I Characterization of the synthesized ox-MWNT-NH${ }_{3}{ }^{+}$(type I) and MWNT-NH ${ }_{3}^{+}$(type $\mathbf{2}$ ) derivatives. (a) Scheme of the synthetic route used for the synthesis of ox-MWNT-NH${ }_{3}{ }^{+}$derivatives. TGA analysis of the ox-MWNT (black line) and ox-MWNT- $\mathrm{NH}_{3}{ }^{+}$(red line). ox-MWNT showed a drop in weight loss at $150^{\circ} \mathrm{C}$, due to the loss in the carboxylic groups introduced on CNT surface, and at $600^{\circ} \mathrm{C}$ due to graphitic-skeleton decomposition. ox-MWNT-NH${ }_{3}{ }^{+}$showed an increase in the weight loss $\left(13 \%\right.$ at $\left.600^{\circ} \mathrm{C}\right)$ due to the introduction of functional groups from the cycloaddition reaction. (b) Scheme of the synthetic route used for the synthesis of MWNT-NH ${ }_{3}{ }^{+}$derivatives. TGA analysis of the pristine MWNT (black line) showed its thermal stability up to $750^{\circ} \mathrm{C}$. MWNT-NH ${ }_{3}^{+}($red line) displayed an increase in the weight loss $\left(15 \%\right.$ at $600^{\circ} \mathrm{C}$ ) due to the introduction of functional groups from the cycloaddition reaction. Length distribution (mean \pm $\mathrm{SD})$ and median, determined from TEM measurements $(n=100)$ are displayed on length distribution histograms.

(DMSO), paraformaldehyde and Ribonuclease A from bovine pancreas RNAse (R6513-50MG) were purchased from Sigma-Aldrich (UK). $0.2 \mu \mathrm{m}$ filter was from Millipore (UK). Dulbecco's modified Eagle medium (DMEM), Ham's F-12 medium, fetal bovine serum (FBS), penicillin/streptomycin, phosphate buffered saline (PBS), Lipofectamine 2000, propidium iodide $(\mathrm{PI})$, and agarose were purchased from Life Technologies (Invitrogen, UK). 6× Orange Loading Dye was from Fermentas (UK) and $0.3 \times$ GelRed stain was from Biotium (USA). Luciferase assay kit was obtained from Promega Corporation (Madison, WI) and BCA protein assay kit from Thermal Scientific (UK). Noncoding siNEG and ATTO 655-labeled siNEG (antisense sequence, 5'-CAUC GUCGAUCGUAGCGCAA-3', \#4754184; sense sequence,
5'-GCGCUACGAUGGACGAUGGG-3', \#4754185), and siPlk-1 (sequence 5'-CGUUGAUGAAGAAGAU CACdTdT-3') were purchased from Eurogentec (Belgium). The human epithelial lung carcinoma A549 (CCL-185) cell line was from American Type Culture Collection (ATCG). A549-Luc cells were purchased from Caliper (Perkin Elmer). Silencer Luciferase siRNA (siLuc) (AM4629) was obtained from Ambion (USA). All chemical substances and solvents were used without further purification.

\section{Chemical Functionalization of MWNTs}

To prepare ammonium-functionalized oxidized multiwalled carbon nanotubes (ox-MWNTs- $\mathrm{NH}_{3}{ }^{+}$) type I, pristine 
MWNTs were firstly oxidized under stirring for $2 \mathrm{~h}$ followed by sonication in a water bath $(20 \mathrm{~W}, 40 \mathrm{kHz})$ for $2 \mathrm{~h}$ in a sulfuric acid/nitric acid solution $(3: 1 v / v, 98$ and 65\%, respectively) at room temperature. Deionized water (DI) was then carefully added and the oxidized MWNTs were filtered (Omnipore GTTP membrane filtration, $0.22 \mu \mathrm{m}$ ), The collected black solid was washed with DI $(300 \mathrm{~mL}), 5 \% \mathrm{NaOH}$ solution $(300 \mathrm{~mL})$ and methanol $(200 \mathrm{~mL})$ yielding $42 \mathrm{mg}$ of ox-MWNT. ox-MWNTs- $\mathrm{NH}_{3}{ }^{+}$(type I) was subjected to the 1,3 dipolar cycloaddition reaction (16). In brief, ox-MWNT (20 mg) were suspended in $20 \mathrm{ml}$ of dimethylformamide (DMF) and sonicated for $10 \mathrm{~min}$. After sonication, the tertiary-butyloxycarbonyl (Boc)-protected amino acid (150 mg, $0.51 \mathrm{mmol}$ ) (Fig. 1a) and paraformaldehyde (18.5 mg, 0. $51 \mathrm{mmol}$ ) were added stepwise $(30 \mathrm{mg}$ amino acid and $3.7 \mathrm{mg}$ formaldehyde every $24 \mathrm{~h}$ ), and the mixture was heated at $125^{\circ} \mathrm{C}$ for 5 days. Multiple centrifugation steps (1811 g, $10 \mathrm{~min})$ were carried out to separate the unreacted MWNTs (remain suspended) from the $f$-MWNTs, which were then filtered through a $0.2 \mu \mathrm{m}$ PTFE filter and the collected black solid was washed with $100 \mathrm{ml}$ of dimethylformamide (DMF) and methanol yielding $18 \mathrm{mg}$ of ox-MWNT-NHBoc. For Boc deprotection, $15 \mathrm{mg}$ of ox-MWNT-NHBoc were suspended in $10 \mathrm{ml} \mathrm{DMF}$, and sonicated for $10 \mathrm{~min}$. After sonication, $10 \mathrm{ml}$ of trifluoroacetic acid (TFA) were added to the reaction mixture. The solution was stirred for $24 \mathrm{~h}$ and the $f$-MWNTs were filtered through a $0.2 \mu \mathrm{m}$ PTFE filter. The collected black solid was washed with $10 \mathrm{ml}$ of DMF and methanol yielding $13 \mathrm{mg}$ of ox-MWNT-NH${ }_{3}{ }^{+}$(type I) (thermogravimetric analysis (TGA) mass loss of $13 \%, 193 \mu \mathrm{mol}$ functional groups/g MWNTs). ox-MWNTs- $\mathrm{NH}_{3}{ }^{+}$were dispersed in $5 \%$ dextrose at concentrations of $100 \mu \mathrm{g} / \mathrm{mL}$ and $1 \mathrm{mg} / \mathrm{mL}$, affording $\mathbf{I}-\mathbf{L}$ and $\mathbf{I}-\mathbf{H}$, respectively.

Ammonium-functionalized multiwalled carbon nanotubes $\left(\mathrm{MWNT}_{\mathrm{s}} \mathrm{NH}_{3}{ }^{+}\right.$, type 2 ) were prepared directly from pristine MWNTs following the 1,3 dipolar cycloaddition reaction asdescribed above, without prior oxidation (Fig. 1b). The Boc group was subsequently deprotected using the same protocol shown for type I derivative, affording MWNTs- $\mathrm{NH}_{3}{ }^{+}$(TGA mass loss of $15 \%, 529 \mu \mathrm{mol}$ functional groups/g MWNTs). MWNTs- $\mathrm{NH}_{3}{ }^{+}$(type 2 ) were dispersed in $5 \%$ dextrose at concentrations of $100 \mu \mathrm{g} / \mathrm{mL}$ and $1 \mathrm{mg} / \mathrm{mL}$, affording $\mathbf{2}-\mathbf{L}$ and $\mathbf{2}-\mathbf{H}$, respectively.

\section{Thermogravimetric Analysis}

MWNT samples were loaded in platinum pans and the TGA analysis was performed using the TGA Q500 (TA instruments, USA). Initially the analysis was carried out isothermally in nitrogen atmosphere at $100^{\circ} \mathrm{C}$ for $20 \mathrm{~min}$ followed by a controlled increase in temperature at a rate of $10^{\circ} \mathrm{C} / \mathrm{min}$ to reach a maximum of $1000^{\circ} \mathrm{C}$ at the end of the analysis.

\section{Length Analysis by Transmission Electron Microscopy (TEM)}

Length distribution histograms were obtained by measuring nanotube length from transmission electron microscopy images $(\mathcal{N}=100)$.

\section{Formulation of Cationic Liposomes and $f$-MWNTs-Liposome Hybrids}

Liposomes, $\mathbf{L}$, were prepared by lipid film hydration method followed by sonication. Briefly, DOTAP and cholesterol (2:1 molar ratio) were dissolved in chloroform:methanol $(4: 1 \mathrm{v} / \mathrm{v})$, the organic solvent was evaporated in a rotary evaporator (Buchi, Switzerland) under vacuum at $40^{\circ} \mathrm{C}$ for $30 \mathrm{~min}$ and then flushed with a $\mathrm{N}_{2}$ stream to remove any residual traces of organic solvent. The dried lipid film was hydrated with $1 \mathrm{ml}$ of $5 \%$ dextrose and multilamellar vesicles (MLV) were reduced in size by sonication for $2 \mathrm{~min}$. Small unilamellar vesicles (SUV) were left for at least $2 \mathrm{~h}$ to anneal before being used or stored at $4^{\circ} \mathrm{C}$ under $\mathrm{N}_{2}$. For the preparation of hybrids I-L, 2-L and $\mathbf{I}-\mathbf{H}, \mathbf{2}-\mathbf{H}$, the same protocol was used, but instead of hydrating the lipid film with $5 \%$ dextrose, solutions of $100 \mu \mathrm{g} / \mathrm{ml}$ and $1 \mathrm{mg} / \mathrm{ml}$ of $f$-MWNTs in $5 \%$ dextrose were used, affording hybrids with $f$-MWNT:lipid mass ratio of 5.3\%:94.7 and 35.8\%:64.2\%, respectively. The final lipid concentration was $2 \mathrm{mM}$. Size reduction was achieved by bath sonication for $2 \mathrm{~min}$.

\section{Electron Microscopy Imaging of the Hybrids}

Low voltage STEM imaging was carried out on the FEI extreme high resolution Magellan $400 \mathrm{~L} \mathrm{SEM} \mathrm{running} \mathrm{at} 20 \mathrm{kV}$ fitted with a second generation FEI retractable STEM detector. This detector has multiple segments that allow capture of different signals simultaneously. The central part of the detector collects electrons at a normal angle producing a conventional bright field images. High angle annular dark field (HAADF) images are produced from scattered electrons that are collected on an annular dark field detector at higher angles; the intensity in HAADF images is directly related to the atomic number of the elements within the sample. Samples were sonicated and dispersed in 5\% dextrose and placed dropwise onto a holey carbon copper support grid for STEM observation.

\section{Fluorescence Labelling of Cationic Liposomes and $f$-MWNTs-Liposome Hybrids}

Fluorescently labelled liposomes and hybrids were prepared for fluorescence spectroscopy studies and in vitro cell uptake studies. Nile Red (lipid probe), emitting in the red region, is a hydrophobic fluorescent dye which was incorporated in the 
lipid bilayer of liposomes. Nile Red (NR) was added to the lipid mixture at a ratio of 1:300 dye:lipid molecules and formulations were prepared as described above. Formulations were kept protected from light.

\section{Physico-Chemical Characterization of Cationic Liposomes and f-MWNTs-Liposome Hybrids}

Measurement of the hydrodynamic diameter and Zetapotential of the prepared formulations were performed using dynamic light scattering (DLS) with a Nanosizer ZS series (Malvern Instruments, Southborough, MA). Disposable polystyrene cells and disposable plain folded capillary Zeta cells were used. Suspensions were diluted in deionized water and measurements were performed at $25^{\circ} \mathrm{C}$. Electrophoretic mobility was used to calculate the $\zeta$-potential using the Helmholtz-Smoluchowski equation. The hydrodynamic size was presented as the average value of 20 runs, with triplicate measurements within each run.

\section{Electrophoretic Mobility Shift Assay}

The ability of $f$-MWNTs-liposome hybrids to complex siRNA was determined by GelRed displacement assay and agarose gel electrophoresis. Complexes were prepared by mixing $0.25 \mu \mathrm{g}(25 \mu \mathrm{l})$ siNEG in 5\% dextrose with an equal volume of DOTAP:chol liposomes or $f$-MWNTs-liposome hybrids at different Nitrogen $(\mathrm{N})$ / Phosphate $(\mathrm{P})$ molar ratios. For example, $0.25 \mu \mathrm{g}$ siRNA: $0.7011 \mu \mathrm{g}$ DOTAP:Chol is equivalent to $1: 1 \mathrm{~N} / \mathrm{P}$ ratio. The positive charge contribution from $f$ MWNT was minimal so that the $f$-MWNT mass was not taking into account when calculating the $\mathrm{N} / \mathrm{P}$ ratio. For comparison, $0.25 \mu \mathrm{g}$ of siRNA was also mixed with $f$-MWNTs at equivalent ratios used in the hybrid. Complexes were incubated at room temperature for $30 \mathrm{~min}$ to allow complete formation. Naked non-coding siRNA (siNEG, $0.25 \mu \mathrm{g}$ ) was included for comparison. After staining with $6 \times$ Orange Loading Dye, the suspensions were loaded onto $1 \%(w / v)$ agarose containing $0.3 \times$ GelRed stain, followed by electrophoresis in $0.5 \times$ Tris-Borate-EDTA buffer (SigmaAldrich, UK) at $80 \mathrm{mV}$ for $50 \mathrm{~min}$. The gels were then visualized under UV light using ChemiDoc MP system (BioRad, UK).

\section{Nile Red Fluorescence Spectroscopy}

NR fluorescence spectroscopy was used to study the interaction between the $f$-MWNTs and cationic liposomes in the formed hybrids. The fluorescence and emission spectra of Nile Red was measured using FLUOstar Omega (BMG labtech, UK) plate reader at excitation and emission wavelengths of $544 \mathrm{~nm}$ and $610-620 \mathrm{~nm}$, respectively. A calibration curve using DOTAP:cholesterol liposome incorporating
Nile Red (L) at a ratio of 1:300, dye:lipid molar ratio was established to confirm that fluorescence values were within the linear range of the instrument (data not shown). Different amounts of liposomes were used so that NR concentrations ranged from 0 to $1 \mu \mathrm{g} / \mathrm{ml}$. Formulations were prepared as described above. This protocol was referred to as the "encapsulation protocol". To exclude the quenching effect of $f$-MWNTs on the dye, control samples were prepared where NR was mixed with $f$-MWNTs in $5 \%$ dextrose (quenching control). Another control group was included in this experiment where $f$-MWNTs were mixed just before measurement with the preformed liposomes containing NR, at equivalent concentrations used in the encapsulation protocol. This protocol was referred to as the "mixing protocol". For NR fluorescence measurements, $20 \mu \mathrm{l}$ of each formulation (quenching control, encapsulation or mixing protocols) were diluted $5 \times$ to a final volume of $100 \mu \mathrm{l}$, yielding final lipid and NR concentrations of $0.6 \mathrm{mM}$ and $0.625 \mu \mathrm{g} / \mathrm{ml}$, respectively.

\section{In Vitro siRNA Stability in Serum}

The extent of enzymatic degradation mediated by serum nucleases was analyzed by incubation of non-coding siRNA (siNEG) complexed with formulations $\mathbf{L}$ and hybrids I-L, I$\mathbf{H}, \mathbf{2}-\mathbf{L}$, and $\mathbf{2}-\mathbf{H}$ with FBS. The complexes were prepared as described above, at N/P charge ratio of 4:1 as siRNA was fully complexed at this ratio as determined by the electrophoretic mobility shift assay. After complexation, FBS $(5 \mu \mathrm{l})$ was added to the complexes at a final concentration of $50 \%(v / v)$ and samples were incubated at $37^{\circ} \mathrm{C}$ for $1 \mathrm{~h}$ or $4 \mathrm{~h}$. To keep the same volume and incubation time as that used in the heparin competition assay, $5 \mu \mathrm{l}$ of $5 \%$ dextrose were added to each sample and samples were further incubated at $37^{\circ} \mathrm{C}$ for $10 \mathrm{~min}$. After incubation, the reaction was terminated by adding EDTA to a final concentration of $50 \mathrm{mM}$. Nonincubated naked siRNA $(0.25 \mu \mathrm{g})$ or naked siRNA $(0.25 \mu \mathrm{g})$ co-incubated with FBS $(50 \% v / v)$ and EDTA $(50 \mathrm{mM})$ were used as negative and positive controls, respectively. Samples were electrophoresed under the conditions described above.

\section{In Vitro siRNA Protection Against RNAse}

The siRNA (siNEG) protection ability of formulations $\mathbf{L}$ and hybrids I-L, I-H, 2-L, and $\mathbf{2}-\mathbf{H}$ from RNAse was assessed. The complexes at $\mathrm{N} / \mathrm{P}$ charge ratio of 4:1 were prepared as described above. After complexes were formed, RNAse $(5 \mu \mathrm{l})$ was added to the complexes at a final concentration of $0.4 \mu \mathrm{g} / \mathrm{ml}$ and samples were incubated at $37^{\circ} \mathrm{C}$ for $10 \mathrm{~min}$ or $1 \mathrm{~h}$. To keep the same volume and incubation time as that used in the heparin competition assay, $5 \mu \mathrm{l}$ of $5 \%$ dextrose was added to each sample and samples were further incubated at $37^{\circ} \mathrm{C}$ for $10 \mathrm{~min}$. After incubation, the reaction was terminated by adding EDTA to a final concentration of $50 \mathrm{mM}$. Non- 
incubated naked siRNA $(0.25 \mu \mathrm{g})$ or naked siRNA $(0.25 \mu \mathrm{g})$ co-incubated with RNAse $(0.4 \mu \mathrm{g} / \mathrm{ml})$ and EDTA $(50 \mathrm{mM})$ were used as negative and positive controls, respectively. Samples were electrophoresed under the conditions described above.

\section{Heparin Competition Assay}

A heparin competition assay was performed to dissociate siRNA (siNEG) complexes in order to examine the siRNA integrity following incubation either with FBS or with RNAse. Following incubation with either FBS or RNAse, heparin was added to each sample to a final concentration of $50 \mathrm{IU} / \mathrm{ml}$ and samples were further incubated at $37^{\circ} \mathrm{C}$ for $10 \mathrm{~min}$. After incubation, the reaction was terminated by adding EDTA to a final concentration of $50 \mathrm{mM}$. Nonincubated naked siRNA $(0.25 \mu \mathrm{g})$ or naked siRNA $(0.25 \mu \mathrm{g})$ with RNAse $(0.4 \mu \mathrm{g} / \mathrm{ml})$, heparin $(50 \mathrm{IU} / \mathrm{ml})$ and EDTA $(50 \mathrm{mM})$ were used as negative and positive controls, respectively. Samples were electrophoresed under the conditions described above.

\section{Cell Culture}

Epithelial lung carcinoma cells (A549) were cultured in Ham's F-12 supplemented with $10 \%$ FBS, $50 \mathrm{U} / \mathrm{ml}$ penicillin, $50 \mu \mathrm{g} / \mathrm{ml}$ streptomycin, $1 \%$ L-glutamine, at $37^{\circ} \mathrm{C}$ in 5\% $\mathrm{CO}_{2}$. Luciferase expressing A549 cells (A549-Luc) were cultured in Ham's F-12 supplemented with 10\% FBS, $1 \%$ L-glutamine, at $37^{\circ} \mathrm{C}$ in $5 \% \mathrm{CO}_{2}$. Cells were routinely grown in $75 \mathrm{~cm}^{2}$ canted-neck tissue culture flasks and passaged twice a week using $0.05 \%$ Trypsin/ EDTA when reaching $80 \%$ confluency in order to maintain exponential growth.

\section{siRNA Uptake Studies in A549 Cells by Flow Cytometry}

A549 cells were subcultured onto 24 -well plates $\left(5 \times 10^{4}\right.$ cells/ well) for $24 \mathrm{~h}$ prior transfection with liposome/siRNA or hybrid/siRNA complexes. Cells were incubated with complexes in serum-free and antibiotics-free medium for $4 \mathrm{~h}$, after which serum was added to make the final concentration of serum to $10 \%(v / v)$. Cells were allowed to interact with the complexes for $24 \mathrm{~h}$ at $37^{\circ} \mathrm{C}$ in a humidified atmosphere $(5 \%$ $\mathrm{CO}_{2}$ ) incubator. After incubation, cells were washed twice with PBS to remove unbound complexes, trypsinized, centrifuged at $800 \mathrm{~g}$ for $5 \mathrm{~min}$, resuspended in PBS, and analyzed by flow cytometry. Whenever cells were not analyzed immediately, they were fixed in $4 \%(v / v)$ paraformaldehyde in PBS solution and stored at $4^{\circ} \mathrm{C}$. Bivariate and univariate histograms were set using the BD FACSCalibur flow cytometer (Becton Dickinson, Franklin Lakes, NJ, USA) equipped with CellQuest Pro software (BD, USA). Ten thousand events were acquired in the gated cell population of interest. For NRlabelled liposomes or hybrids, cell uptake was assessed by NR fluorescence emission (FL-3 detector) and also by sideward scattering (SSC) as described previously $(17,18)$. Unlabeled non-coding siRNA (siNEG) was used in these experiments. For tracking siRNA internalization, ATTO 655labelled siNEG was used in combination with nonfluorescently labelled $\mathbf{L}$ or the hybrids. ATTO 655-siNEG uptake was measured using FL-4 detector.

\section{Side Scatter Analysis (Complexity) in A549 Cells by Flow Cytometry}

In the bivariate scatter histograms, the sideward scattering (SSC Lin, ordinate) vs. forward scattering histogram (FSC Lin, abscissa) was obtained. The forward scatter and side scatter detectors voltage had to be set in a way that both the negative control (a sample containing cells without any treatment) and the positive control cells (a sample containing cells that were transfected with DOTAP:chol/siRNA complexes) could be visualized inside the scatter dot plot. The population containing cells only was selected to include all the cells being studied and exclude any cell debris. In order not to include $f$ MWNTs in the cell population group, a suspension containing the highest concentration of $f$-MWNTs without any cells was run first to exclude any free CNT from the cell population. The univariate histograms of cell number (counts, ordinate) $v s$. either the sideward scattering intensity (SSC Lin, abscissa) or the fluorescence intensity (FL-3 Log for NR fluorescence or FL-4 Log for ATTO 655 fluorescence, abscissa) were plotted and gated for the cell population. The mean sideward scattering intensity values (SSC Lin) or the mean fluorescence intensity values (FL-3 and FL-4 Log) were recorded and used for comparison.

\section{Luciferase Expression in A549-Luc Cells}

A549-Luc cells were seeded onto 96-well plates $\left(1 \times 10^{4}\right.$ cells/ well) with antibiotics-free F-12 medium $24 \mathrm{~h}$ prior transfection. The next day, siLuc or siNEG $(10 \mu \mathrm{l})$ was complexed with an equal volume of formulations (L or $\mathbf{I}-\mathbf{L})$ in $5 \%$ dextrose at $\mathrm{N} / \mathrm{P}$ charge ratio of $4: 1$. Following incubation at room temperature for $30 \mathrm{~min}$, complexes were diluted in serum-free and antibiotics-free medium and added to the cells to achieve a final siRNA concentration of $80 \mathrm{nM}$. After $4 \mathrm{~h}$, serum was added to reach $10 \%(v / v)$ final serum concentration. At $48 \mathrm{~h}$ post-transfection, cell lysate was prepared and luciferase expression was assayed with a Luciferase assay kit using a luminometer FLUOstar Omega (BMG labtech, UK) plate reader. Briefly, cells were washed with PBS before addition of $60 \mu \mathrm{l}$ of lysis buffer. Two freeze-thaw cycles were performed to ensure complete cell lysis and cells were directly centrifuged in the 96-well plate for $30 \mathrm{~min}$ at high speed and $4^{\circ} \mathrm{C} .20 \mu \mathrm{l}$ of 
the supernatant containing proteins was mixed with $100 \mu \mathrm{l}$ of luciferase assay reagent and then luminescence signals were recorded. $20 \mu \mathrm{l}$ of the lysate was used to determine the protein concentration using a BCA protein assay kit, following manufacturer's instructions. The Luciferase activity was calculated as RLU/mg protein. Values were normalized to siNEG transfected cells.

\section{Biological Activity of siPLKI in A549 Cells With MTT Assay}

In order to assess the functionality of siRNA delivered by $\mathbf{L}$ or $\mathbf{I - L}$, a functional siRNA (siPLK1) was used and toxicity induced by PLK1-specific gene silencing was determined. A549 cells were seeded onto 96 -well plates $\left(1 \times 10^{4}\right.$ cells/well $)$ with antibiotics-free F-12 medium $24 \mathrm{~h}$ prior transfection. The next day, siPLK1 or siNEG $(10 \mu \mathrm{l})$ was complexed with an equal volume of the formulation $(\mathbf{L}$ or $\mathbf{I}-\mathbf{L})$ in $5 \%$ dextrose at N/P charge ratio of 4:1. Lipofectamine 2000 was used as a positive control for the transfection, whereas cells treated with $10 \%$ DMSO were used as a cytotoxic control for the MTT assay. Following incubation at room temperature for $30 \mathrm{~min}$, complexes were diluted in serum-free and antibiotics-free medium and added to the cells, yielding a final siRNA concentration of $80 \mathrm{nM}$. After $4 \mathrm{~h}$, serum was added to reach $10 \%$ $(v / v)$ final serum concentration. At $48 \mathrm{~h}$ post transfection, MTT assay was performed. Cells were incubated with MTT solution at $0.5 \mathrm{mg} / \mathrm{ml} \mathrm{MTT} \mathrm{final} \mathrm{concentration} \mathrm{for} 3 \mathrm{~h}$. Media was then removed and the formazan produced was dissolved in $200 \mu \mathrm{l}$ DMSO and absorbance was read in a FLUOstar Omega (BMG labtech, UK) plate reader at $560 \mathrm{~nm}$. Results were expressed as the percentage of cell viability (mean \pm SD) and normalized to siNEG transfected grow of each carrier. The following equation was used: \% cell viability $=($ A560 $\mathrm{nm}$ of siPLK1 transfected cells $/$ A560 nm of siNEG transfected cells $) \times 100$.

\section{Apoptosis Measurement and Sub-GI Analysis with Flow Cytometry}

In order to assess the effect of combining PLK1 downregulation with Dox toxicity using the different formulations on cell apoptosis (as a therapeutic endpoint), sub-G1 analysis was carried out by flow cytometry. MWNT has been reported to interfere with MTT assay so it was avoided here (19). A549 cells were seeded onto 24 -well plates $\left(5 \times 10^{4}\right.$ cells/well) for $24 \mathrm{~h}$ prior transfection. The next day, siPLK1 or siNEG was complexed with equal volume of formulation (L or I-L) in $5 \%$ dextrose at N/P charge ratio of $4: 1$. An equal volume of Dox was added to each complex and allowed to complex with MWNT for $30 \mathrm{~min}$ at room temperature, yielding a final Dox concentration of $1 \mu \mathrm{M}$. Cells were then incubated with the complexes (with or without Dox) in antibiotics-free medium for $48 \mathrm{~h}$ at $37^{\circ} \mathrm{C}$ in a humidified atmosphere $\left(5 \% \mathrm{CO}_{2}\right)$ incubator. After incubation, both attached cells and those in suspension were harvested, washed in PBS and fixed with cold $70 \%(v / v)$ ethanol for at least $1 \mathrm{~h}$, at $4^{\circ} \mathrm{C}$. Following fixation, cells were centrifuged at $850 \mathrm{~g}$ for $5 \mathrm{~min}$ at $4^{\circ} \mathrm{C}$ to remove ethanol. The cells were then resuspended in PBS:citrate buffer $(1: 1 v / v)$ and incubated for $5 \mathrm{~min}$ at $37^{\circ} \mathrm{C}$. After incubation, supernatant was removed by centrifugation at $850 \mathrm{~g}$ for $5 \mathrm{~min}$ at $22^{\circ} \mathrm{C}$. The cells were then resuspended in PBS solution containing propidium iodide $(50 \mu \mathrm{g} / \mathrm{ml})$ and RNAse $(100 \mu \mathrm{g} / \mathrm{ml})$ and incubated in the dark for $30 \mathrm{~min}$ at $37^{\circ} \mathrm{C}$. Cells were then analyzed by BD FACSCalibur flow cytometer. The percentage of events in the sub-G1 phase of the cell cycle histogram (10,000 events) was determined using CellQuest Pro software (BD, USA).

\section{Statistical Analysis}

Data are presented as means \pm standard deviation (SD). The statistical analysis was performed using one-way ANOVA followed by Tukey's multiple comparison test (GraphPad Prism 5.0 software). Differences between groups were considered significant for $* p<0.05$, highly significant for $*^{*} p<0.01$ and extremely significant for $* * * p<0.001$.

\section{RESULTS}

\section{Physicochemical Characterization of $f$-MWNTs-Liposome Hybrids}

Initially the length distribution of the $f$-MWNT was performed from TEM images $(n=100)$. The length of the pristine MWNT is provided by the supplier $(0.5-2 \mu \mathrm{m})$. In case of oxidized MWNT (type 1), pristine MWNT is subjected to acid treatment step which results in shortening to $277.5+/-152.93 \mathrm{~nm}$ (20). In case of type 2 where no oxidation step was performed, the length was $455.5+/-185.42 \mathrm{~nm}$. Cycloaddition reaction is not expected to reduce the length of MWNT (15), however, the centrifugation and filtration steps during functionalization leads to exclusion of longer tubes.

TGA is a technique widely used to characterize materials with different thermal stabilities. It was therefore used here to quantify the degree of chemical functionalization with organic functional groups (Fig. 1). In Fig. la, ox-MWNT- $\mathrm{NH}_{3}{ }^{+}$(type $\mathbf{I}$, red line) showed a drop in weight loss at $150^{\circ} \mathrm{C}$, due to the loss in the carboxylic groups introduced on CNT surface, and at $600^{\circ} \mathrm{C}$ due to graphitic-skeleton decomposition. oxMWNT- $\mathrm{NH}_{3}{ }^{+}$showed an increase in the weight loss of $13 \%$ at $600^{\circ} \mathrm{C}$ confirming the introduction of functional groups from the cycloaddition reaction. Pristine MWNTs were thermally stable up to $750^{\circ} \mathrm{C}$ (Fig. 1b, black line). MWNT- $\mathrm{NH}_{3}{ }^{+}$(type 2) (Fig. 1b, red line) displayed an increase 
in the weight loss of $15 \%$ at $600^{\circ} \mathrm{C}$ also due to the introduction of functional groups from the cycloaddition reaction. The degree of chemical functionalization for ox-MWNT- $\mathrm{NH}_{3}{ }^{+}$ (type I) and MWNT- $\mathrm{NH}_{3}{ }^{+}$(type 2) was calculated as $193 \mu \mathrm{mol}$ and $529 \mu \mathrm{mol}$ functional groups/g MWNTs, respectively.

Physicochemical characterization of the four hybrids was also carried out to determine their feasibility as co-delivery systems. The composition, hydrodynamic diameter, polydispersity index (PDI) and $\zeta$-potential of the different formulations tested are summarized in Table I. The hydrodynamic diameters of all formulations tested were, on average, less than $200 \mathrm{~nm}$, with all $f$-MWNT-liposome hybrids showing greater sizes compared to cationic liposome $(\mathbf{L})$. The reported hydrodynamic diameters of all hybrids were surprisingly smaller than the length of the corresponding MWNT (Fig. 1). An explanation for this could be the fact that the hydrodynamic diameter (Z-average) reflects a combined overall value and not the magnitude of just one dimension of the nanocarriers (21). Therefore, since the $f$-MWNTs are not spherical particles and their diameter is much smaller (20-30 nm) compared to their length, the hydrodynamic diameter of hybrids reflects a combined outcome of the two dimensions. Although DLS is not the best technique to measure the size of CNTs, we used this method as PDI values were indicative of the size distribution. PDI of hybrids $\mathbf{2}-\mathbf{L}$ and $\mathbf{2}-\mathbf{H}$, formed with MWNT$\mathrm{NH}_{3}{ }^{+}$, was greater than for those formed with ox-MWNT$\mathrm{NH}_{3}{ }^{+}$(hybrids $\mathbf{I}-\mathbf{L}$ and $\mathbf{I}-\mathbf{H}$ ). This was expected as the GNTs that form hybrid type $\mathbf{2}$ are longer than the CNTs that form hybrid type $\mathbf{I}$.

All formulations displayed positive $\zeta$-potential values, ranging from 32 to $52 \mathrm{mV}$, comparable to that of cationic liposomes $(48 \mathrm{mV})$.

\section{Morphological Studies}

The morphology of the liposomes and hybrids was studied by low voltage STEM at $20 \mathrm{kV}$. This operating voltage allowed us to study the structure of these beam sensitive samples avoiding the knock-on damage occurring at higher operating voltages $(>80 \mathrm{kV})$.
Sample $\mathbf{L}$ showed round features of 60-200 $\mathrm{nm}$ in diameter in agreement with the hydrodynamic diameter of the cationic liposomes (Fig. 2). The cationic liposomes were found in micron-size agglomerates most probably formed upon solvent drying during the TEM grid preparation.

STEM images of sample IH (Fig. 2 and S1) showed a clear interaction of the cationic liposomes with MWNTs. Round features with faint contrast corresponding to the cationic liposomes were present close to the ox-MWNT- $\mathrm{NH}_{3}{ }^{+}$and even embedding them.

AFM height images, in 2D and 3D for $\mathbf{L}$ and $\mathbf{I}-\mathbf{H}$ are shown in Fig. S2. Cross-section analysis exhibited height of 5-10 nm for both samples indicating the collapse of the liposomes on the mica surface. Samples $\mathbf{I}-\mathbf{H}$ showed more heterogeneous structures compared to liposome sample.

\section{siRNA Binding}

The ability of the $f$-MWNT-liposome hybrids to complex nucleic acids and the optimum N/P ratio required for complexation were determined using an electrophoretic mobility assay across a range of $\mathrm{N}: \mathrm{P}$ charge ratios from $0.5: 1$ to $8: 1$. The siRNA complexation profile of $\mathbf{I}-\mathbf{L}, \mathbf{I}-\mathbf{H}, \mathbf{2}-\mathbf{L}$, and $\mathbf{2}-\mathbf{H}$ is shown in Fig. 3. DOTAP:chol liposomes (formulation L) and $f$-MWNTs were used as controls. DOTAP:chol liposomes and hybrids I-L, I-H, 2-L, and $\mathbf{2}-\mathbf{H}$ present a similar complexation profile, where full siRNA complexation was achieved at $\mathrm{N} / \mathrm{P}$ ratio of $4: 1$, demonstrated by the absence of free siRNA band and concomitant increased fluorescence in the well. These results are in agreement with those obtained for $\zeta$ potential measurements, where the overall surface charge of the hybrids remained unaltered upon hybridization with $f$ MWNTs. Therefore, it was expected that carriers ability to form complexes with siRNA would not be affected upon hybrid formation. This confirms the suitability of using the hybrids for siRNA delivery. $f$-MWNTs complexation profile in Fig. 3 represents the equivalent $f$-MWNTs amount present in the hybrid at the $\mathrm{N} / \mathrm{P}$ ratios studied, which as expected afforded $\mathrm{N} / \mathrm{P}$ ratios lower than those required to achieve complexation.

Table I Physico-chemical characterization of the hybrids.

\begin{tabular}{|c|c|c|c|c|c|}
\hline Formulation & $\begin{array}{l}\text { CNT final concentration } \\
(\mu g / \mathrm{mL})\end{array}$ & $\begin{array}{l}\text { Formulation composition } \\
\text { CNT:total lipid (w/w\%) }\end{array}$ & $\begin{array}{l}\text { Hydrodynamic } \\
\text { diameter } \pm \mathrm{SD}(\mathrm{nm})\end{array}$ & $\begin{array}{l}\text { Polydispersity } \\
\text { index }\end{array}$ & $\begin{array}{l}\zeta \text {-potential } \pm S D \\
(\mathrm{mV})\end{array}$ \\
\hline L & 0 & $0: 100$ & $118.9 \pm 1.83$ & 0.256 & $48 \pm 5.82$ \\
\hline Hybrid I-L & 100 & $5.3: 94.7$ & $185.9 \pm 1.12$ & 0.319 & $52.4 \pm 3.45$ \\
\hline Hybrid I-H & 1000 & $35.8: 64.2$ & $|67| \pm 0.78$. & 0.256 & $47.4 \pm 0.80$ \\
\hline Hybrid 2-L & 100 & $5.3: 94.7$ & $166.4 \pm 2.22$ & 0.411 & $31.9 \pm 1.80$ \\
\hline Hybrid 2-H & 1000 & $35.8: 64.2$ & $198.9 \pm 4.15$ & 0.354 & $51.8 \pm 1.87$ \\
\hline
\end{tabular}


Fig. 2 Morphological examination of $\mathbf{L}$ and $\mathbf{I}-\mathbf{H}$. Low voltage STEM images of liposomes $(\mathbf{L})$ and the I-H hybrid.
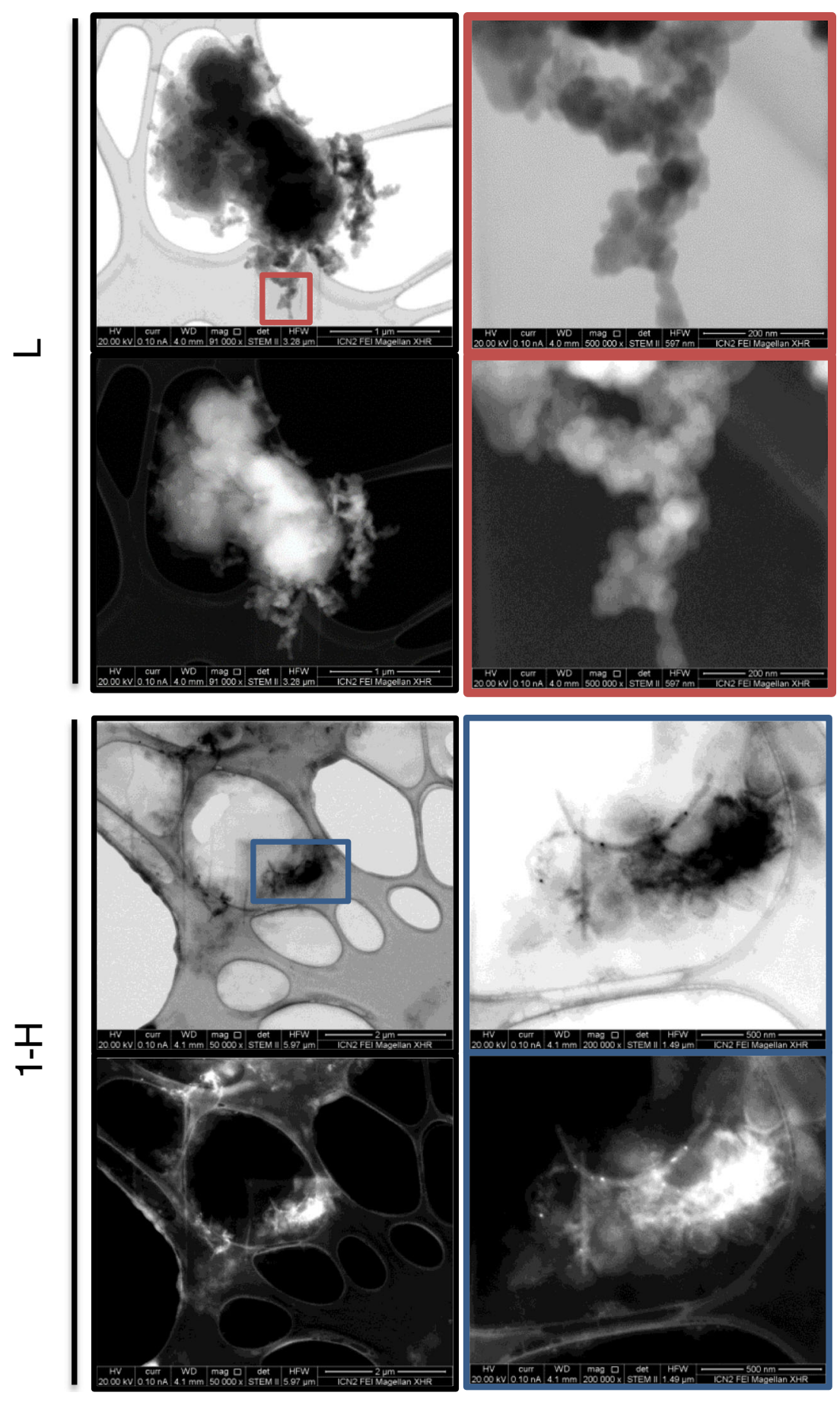

\section{Nile Red Fluorescence Spectroscopy}

Nile Red (NR) is a benzophenoxazone dye commonly used to study the interaction with lipid bilayers (22). It is hypothesized that when NR exists in non-polar environment, its fluorescence increases. A reduction in NR fluorescence in the hybrid form compared to liposome can be attributed to interactions between $f$-MWNT and liposome. The following groups were included in the study (Fig. 4): (i) NR mixed with $f$-MWNTs in
$5 \%$ dextrose (quenching controls), (ii) NR incorporated into liposome during its formation then mixed with $f$-MWNT just before measurement (mixing control), (iii) NR incorporated into $f$-MWNT-liposome hybrid during its formation (encapsulation protocol). NR fluorescence intensities were monitored over a period of $24 \mathrm{~h}$.

After $24 \mathrm{~h}$ incubation, $f$-MWNT I-L and 2-L (Fig. 4a), showed a reduction of 22.8 and $9 \%$ in NR fluorescence intensity, respectively, compared to NR in 5\% dextrose. When 

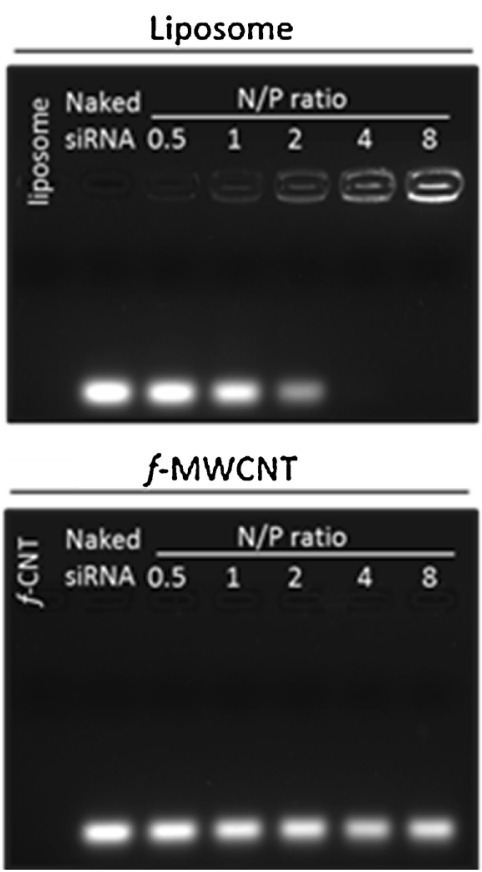

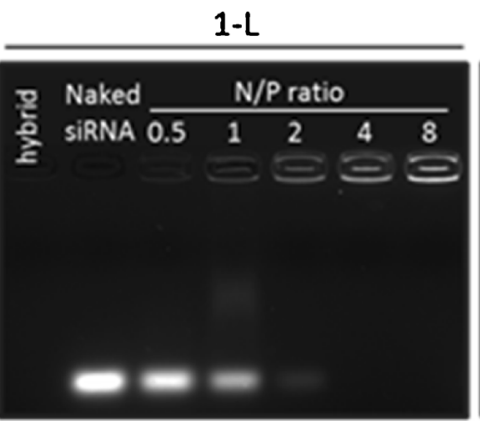

$1-H$

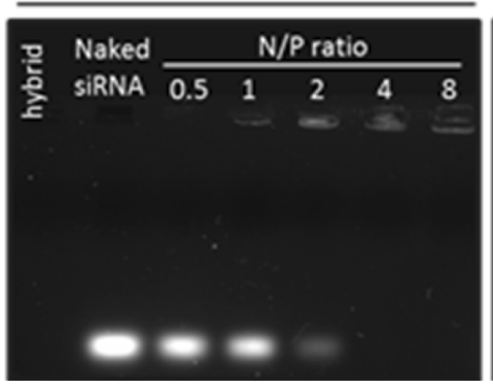

$2-L$

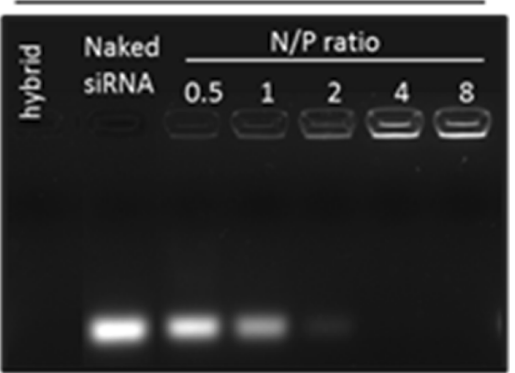

$2-\mathrm{H}$

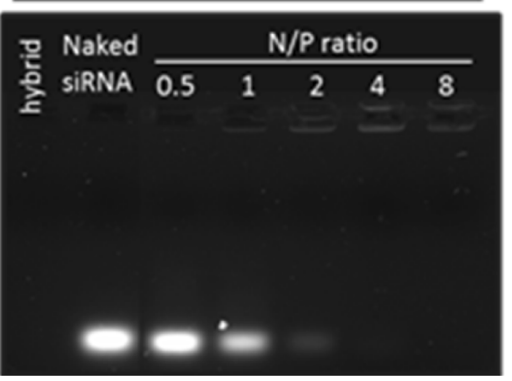

Fig. 3 Electrophoretic mobility shift assay of the different formulations complexed with siRNA at various N/P ratios. Charge ratios are calculated for a fixed siRNA amount of $0.25 \mu \mathrm{g}$. Lane I - naked siRNA $(0.25 \mu \mathrm{g})$, Lanes $2-6$ - complexes at different N/P ratios.
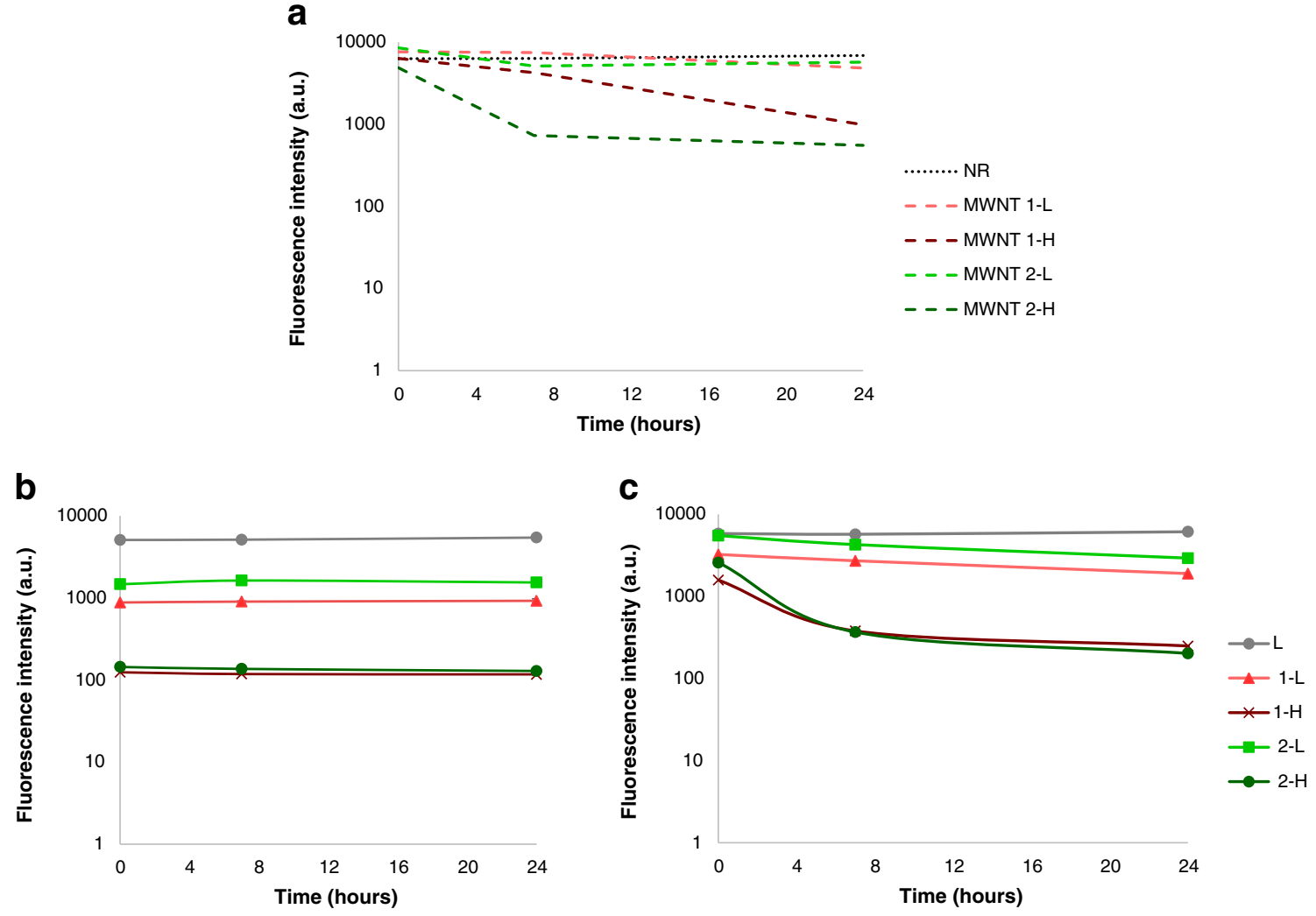

Fig. 4 NR fluorescence spectroscopy for (a) NR mixed with f-MWNTs in 5\% dextrose (quenching controls), (b) NR used in encapsulation protocol (the lipid film containing NR is hydrated with the f-MWNTs), (c) NR used in the mixing protocol (f-MWNTs were mixed with the pre-formed labelled liposomes) over time. NR fluorescence was detected at excitation and emission wavelengths of $544 \mathrm{~nm}$ and 61 0-620 nm, respectively. Fluorescence intensity values were measured at 0,7 and $24 \mathrm{~h}$. Results were expressed as means $\pm S D, n=3$. 
higher concentrations of $f-\mathrm{MWNT} \mathbf{I}-\mathbf{L}$ and $\mathbf{2}-\mathbf{L}$ were used, mimicking their content in $\mathbf{I}-\mathbf{H}$ and $\mathbf{2}-\mathbf{H}$ hybrids, NR fluorescence reduced by 84.3 and $91.2 \%$, respectively. In case of the hybrids prepared at a ratio of 5.3\% (w/w) (Fig. 4b and c), when incubation time increased from 0 to $24 \mathrm{~h}$, hybrid I-L and $\mathbf{2}-\mathbf{L}$ showed reductions in NR fluorescence intensity by 82.7 and $70.3 \%$, respectively, compared to $\mathbf{L}$. This suggests that both types of $f$-MWNTs exhibited interactions with DOTAP:chol liposomes. NR quenching started to be seen at high $f$ MWNT concentrations (equivalent to $35.8 \% w / w)$ in both $f$ MWNTs and the hybrids ( $>84 \%$ reduction in fluorescence) (Fig. 4b and c). In summary, NR spectroscopy studies suggest that the lower NR fluorescence intensities obtained for the hybrids, of low f-MWNT content, compared to cationic liposomes could be a result of the interaction between $f$-MWNTs and liposomes. Conclusions on interactions could not be made at in $1-\mathrm{H}$ and $2-\mathrm{H}$ due to possibility of quenching.

\section{f-MWNT-Liposome Hybrids Stability Against Serum and RNAse}

The ability of the different f-MWNT-liposome hybrids to protect siRNA from nuclease mediated degradation was evaluated by challenging the siRNA complexes with serum. Naked siRNA, liposome/siRNA and hybrid/siRNA complexes were incubated at $37^{\circ} \mathrm{C}$ in $50 \%$ serum for $1 \mathrm{~h}$ or $4 \mathrm{~h}$. As shown in Fig. 5a, after $4 \mathrm{~h}$ incubation with serum, naked siRNA was almost completely degraded, whereas both liposome and hybrids prolonged siRNA stability in serum at 4:1 N/P ratio. The heparin competition assay further confirmed that complex formation stabilized and protected siRNA in serum (Fig. 5b). The siRNA bands appeared at the same position as the control bands after dissociation of the siRNA/liposome or siRNA/hybrid with comparable intensities. Furthermore, incubation with serum up to $4 \mathrm{~h}$ did not seem to significantly affect the stability of these complexes.

The ability of complexes to protect siRNA against a ribonuclease (RNAse) was conducted by incubation for $10 \mathrm{~min}$ or $60 \mathrm{~min}$ at $37^{\circ} \mathrm{C}$. As shown in Fig. 5c, naked siRNA was completely degraded after 10 min incubation with RNAse, whereas both liposome and hybrids prolonged siRNA stability in RNAse at 4:1 N/P ratio, demonstrated by the fluorescence in the wells. Following siRNA release mediated by heparin, hybrids $\mathbf{I}-\mathbf{L}$ and $\mathbf{2}-\mathbf{L}$ showed siRNA band intensities comparable to that of liposome (L) at the respective incubation times (Fig. 5d). In contrast, the extent of siRNA degradation by RNAse was higher for hybrids prepared with high concentration of $f$-MWNTs, in particular for $\mathbf{2}-\mathbf{H}$ which was unable to protect siRNA from digestion at both time points. It is evident that $\mathbf{2}-\mathbf{H}$ complexes are least stable compared to complexes formed with other hybrids thus, offering lowest siRNA protection against both serum and RNAse. This result is in agreement with NR fluorescence measurements where MWNT$\mathrm{NH}_{3}{ }^{+}$displayed a lower degree of interaction with cationic liposomes compared to ox-MWNT- $\mathrm{NH}_{3}{ }^{+}$. Interestingly,
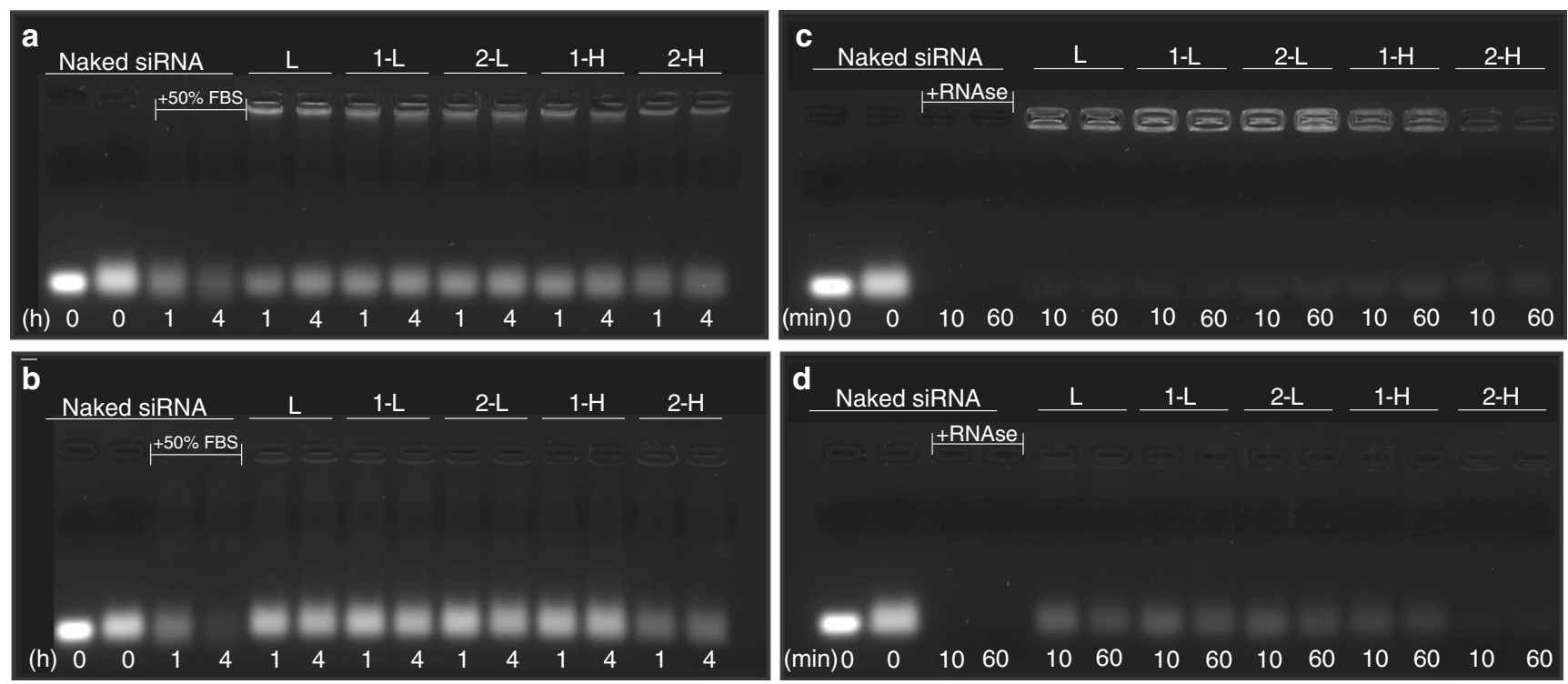

Fig. 5 Agarose gel electrophoresis for complexes subjected to (a) serum; (b) serum followed by heparin competition assay of the different formulations complexed with siRNA at 4:I N/P ratio; (c) RNAse; (d) RNAse followed by heparin competition assay of the different formulations complexed with siRNA at 4: I N/P ratio. The complexes were prepared at a fixed siRNA amount of $0.25 \mu$ g. Complexes were incubated with $50 \%$ ( $/ \mathrm{V}$ ) FBS (I h or $4 \mathrm{~h}$ ) or RNAse (I 0 min or $60 \mathrm{~min}$ ) at $37^{\circ} \mathrm{C}$. For the heparin competition assay, complexes were further incubated for 10 min in the presence of $501 \mathrm{U} / \mathrm{mL}$ heparin. $50 \mathrm{mM} \mathrm{EDTA}$ was used to inactivate serum proteins. In (a), (b) Lanes I and 2 (far left)-naked siRNA or naked siRNA + FBS + heparin + EDTA, respectively. In (c), (d) Lanes I and 2 (far left)- naked siRNA or naked siRNA + RNAse + heparin + EDTA, respectively. 
although all hybrids tested demonstrated similar siRNA complexation profiles, their siRNA protection capacities were different, indicating different stabilities, with the hybrids prepared with the lowest concentration of $f$-MWNTs presenting the most prominent protection ability which was comparable to that of $\mathbf{L}$. As hybrid $\mathbf{2}-\mathbf{H}$ did not appear to be very stable, hybrid type $\mathbf{2}$ was not included in the subsequent experiments. Furthermore, hybrids with lowest $f$-MWNT amount were preferred so next set of experiments was focused on hybrid I-L.

\section{ATTO 655-siRNA and Hybrid Cellular Uptake in A549 Cells}

To study the uptake of complexed hybrids in human A549 monolayers, DOTAP:chol/siRNA or I-L/siRNA complexes were incubated with cells for $24 \mathrm{~h}$. The uptake of the carrier $\mathbf{L}$ or $\mathbf{I}-\mathbf{L}$ ) was monitored by measuring Mean Fluorescence Intensity (MFI) of NR (FL-3) by flow cytometry. Uptake of ATTO 655 labelled-siNEG (emitting at $\lambda_{\mathrm{em}}=684 \mathrm{~nm}$ )/(FL4) allowed siRNA uptake quantification. Side scatter was used to monitor changes in cell complexity reflecting extent of $f$ MWNT uptake as we reported previously. Interestingly, when cells were allowed to interact with the hybrid complexes for $24 \mathrm{~h}$, a significant increase in the sideward-scatter ( $\mathrm{SSC}$ ) by cells was observed compared to the naive (untreated cells) or cells treated with $\mathbf{L}$ complexes (Fig. $6 \mathrm{a}$ and b). In line with the SSC results, cells incubated with I-L/siNEG complexes displayed higher NR fluorescence than those incubated with L/siNEG complexes (Fig. 6c). Fluorescently labelled ATTO 655 siNEG was further employed to assess the siRNA delivery capacities of $\mathbf{I}-\mathbf{L}$ compared to $\mathbf{L}$, and to confirm if differences between the two delivery systems could actually be seen. Figure $6 \mathrm{~d}$ represents MFI (FL-4) of cells incubated with ATTO 655 complexes after $24 \mathrm{~h}$ incubation with cells. A significantly higher siRNA uptake ( $\sim 3$ fold increase) was observed for I-L compared to $\mathbf{L}$, consistent with the results obtained for NR labelled carriers.

\section{Functional Gene Silencing in A549 Cells}

The ability of $f$-MWNT-liposome hybrids to effectively silence a particular gene was studied using two experimental setups that evaluated gene silencing activity. First, A549-Luc cells were transfected with siLuc (siRNA that silence the non-functional Luciferase gene) using $\mathbf{L}$ or $\mathbf{I}-\mathbf{L}$ (Fig. 7a). A comparable level of Luciferase silencing was achieved at $48 \mathrm{~h}$ by the two systems of $48.4 \% \pm$ 11.1 (L) and $49.6 \% \pm 9.6(\mathbf{I}-\mathbf{L})$. Consistent with the results obtained for Luciferase silencing, $\mathbf{L}$ and $\mathbf{I}-\mathbf{L}$ induced comparable levels of PLK1 silencing which resulted in $-20 \%$ cell kill at $48 \mathrm{~h}$ post-transfection (Fig. 7b).
siPLKI and Doxorubicin Co-Delivery to A549 Cells

To assess the feasibility of the new hybrids as co-delivery systems, we next carried out sub-G1 analysis to evaluate cellular apoptosis induced by PLK1 depletion and Dox toxicity using $\mathbf{L}$ and I-L (Fig. 8). A549 cells were incubated with liposomal system $\mathbf{L}$ and I-L complexed with siPLK1 (with or without $1 \mu \mathrm{M}$ Dox) and sub-G1 analysis was performed using flow cytometry after $48 \mathrm{~h}$ of incubation. Cells incubated with $1 \mu \mathrm{M}$ Dox or transfected with siPLK1 as a single agent were used as positive controls. Interestingly, when Dox and siPLK1 were co-delivered to A549 cells, a synergistic effect was achieved with both carriers $\mathbf{L}$ and I-L. More importantly, the synergism between siPLK1 and Dox dual therapy was significantly more pronounced when the two agents were delivered by $\mathbf{I}-\mathbf{L}(40.3 \% \pm 1.5$ apoptotic cells $)$ compared to system $\mathbf{L}(26.0 \% \pm 4.3$ apoptotic cells $)$.

\section{DISCUSSION}

Several studies reported that the co-delivery of a chemotherapeutic agent and siRNA significantly enhances the cytotoxicity to cancer cells and has great potential to overcome the drug resistance. This approach also reduces the side effects of chemotherapy as lower dosages of the cytotoxic drug are needed to achieve similar therapeutic effect. However, reports on delivering siRNA simultaneously with a conventional anticancer drug to cancer cells for enhanced chemotherapy efficacy have been scarce, due to the lack of efficient co-delivery methods $(23,24)$. Due to PLK1 altered expression in a wide range of cancers and to its roles in cancer progression, it was the chosen target to siRNA therapy. The cationic nature of liposomes is essential to efficiently complex the negatively charged siRNA via electrostatic interactions and for interaction with the negatively charged cell membrane.

Recently, Karchemski et al. reported the use of CNTsliposomes conjugate in an attempt of combining the efficient cell uptake of CNTs with the well-known high drug loading capacity of liposomes, which may result in the prevention of potential adverse systemic side effects as lower amount of CNTs are required while enhanced cellular uptake can still be obtained (25). In another study, Miyako et al. successfully developed self-assembled CNT-liposome supramolecular nanotrains, which function as intelligent molecular-transport systems. Utilizing the photothermal property of CNTs and the temperature-responsive property of liposomes, controlled released was achieved by illumination of laser causing the destruction of liposome and therefore the release of its cargo (26). By taking advantage of the highly efficient cellular uptake and siRNA delivery of cationic liposomes and the high drug loading capacity of GNTs and their ability to shuttle cytotoxic drugs into cells, we aimed at formulating $f$-MWNTs-cationic 
Fig. 6 Analysis of cellular uptake of DOTAP:chol liposomes (L) and Ox-MWNT- $\mathrm{NH}_{3}{ }^{+}$-DOTAP:chol hybrid (I-L) and complexed with siRNA in A549 cells using flow cytometry. (a) Bivariate scatter histograms of the sidewardscattering (SSC, Y-axis) versus forward-scattering (FSC, X-axis), (b) Mean sideward-scattering and (c) Mean Fluorescence Intensity of NR labelled $\mathbf{L}$ or $\mathbf{I}-\mathbf{L}$ complexed with non-fluorescent siNEG. (d) Mean Fluorescence Intensity of ATTO 655-labelled siNEG complexed with unlabeled $\mathbf{L}$ or $\mathbf{I}-\mathbf{L}$. Flow cytometry analysis was performed after $24 \mathrm{~h}$ incubation with complexes. Experiments were done in triplicates and expressed as mean $\pm \mathrm{SD}(n=3)$. a
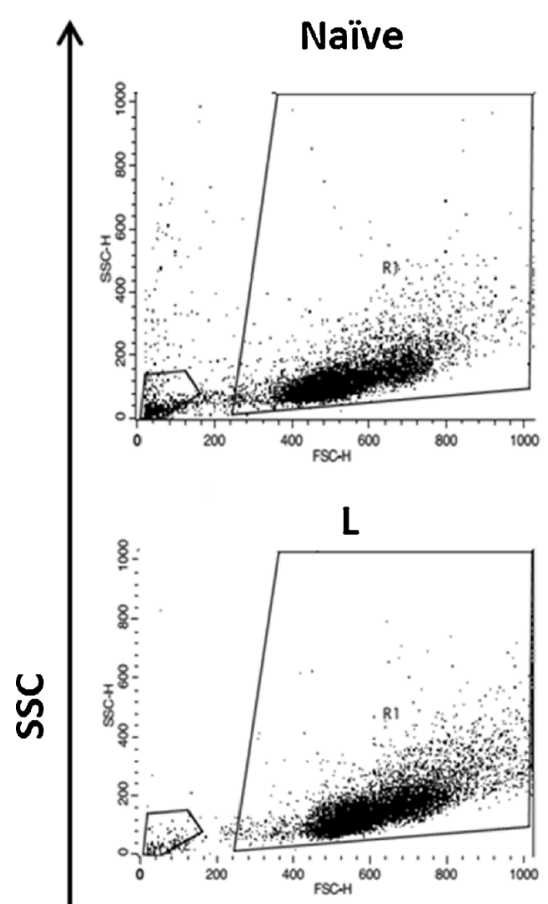

1-L

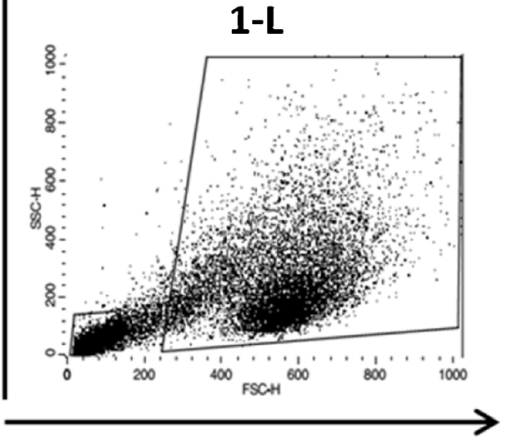

FSC b
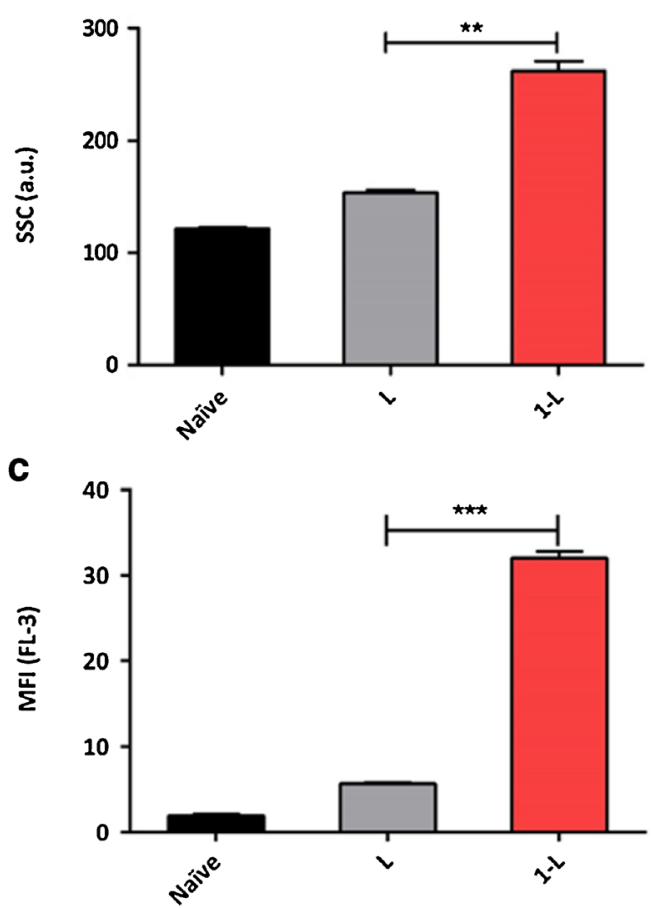

d

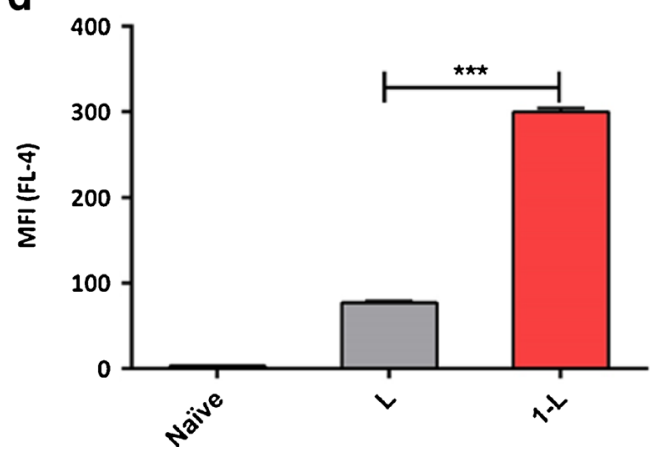

a

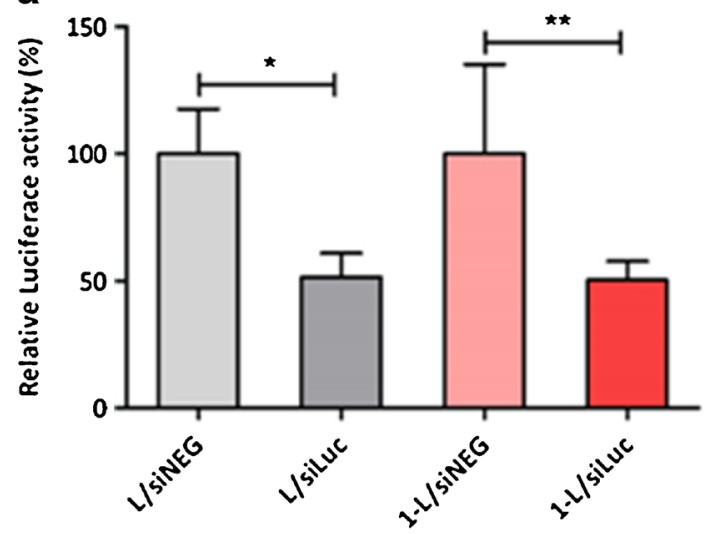

b

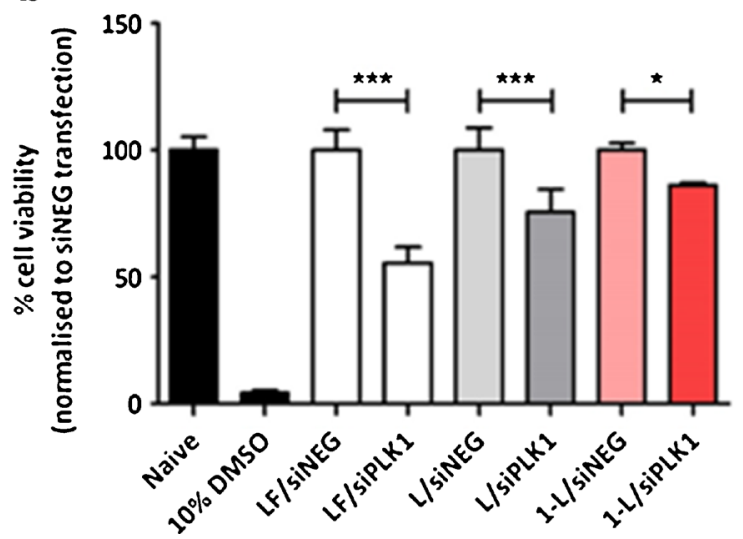

Fig. 7 Analysis of gene silencing efficiency of DOTAP:chol liposomes (L) and ox-MWNT-NH ${ }_{3}{ }^{+}$-DOTAP:chol hybrid (I-L). (a) Relative luciferase activity in A549-Luc cells transfected with $\mathbf{L}$ or $\mathbf{I}-\mathbf{L}$ complexed with non-coding siRNA (siNEG) or siRNA targeting Luciferase (siLuc). Cells were collected for luciferase and BCA assays $48 \mathrm{~h}$ post-transfection. (b) Cell viability (\%) determined by MTT assay after $48 \mathrm{~h}$ transfection with $\mathbf{L}$ and $\mathbf{I}$-L complexed with siNEG or siPLKI . Results were expressed as mean $\pm \mathrm{SD}(n=5)$. 


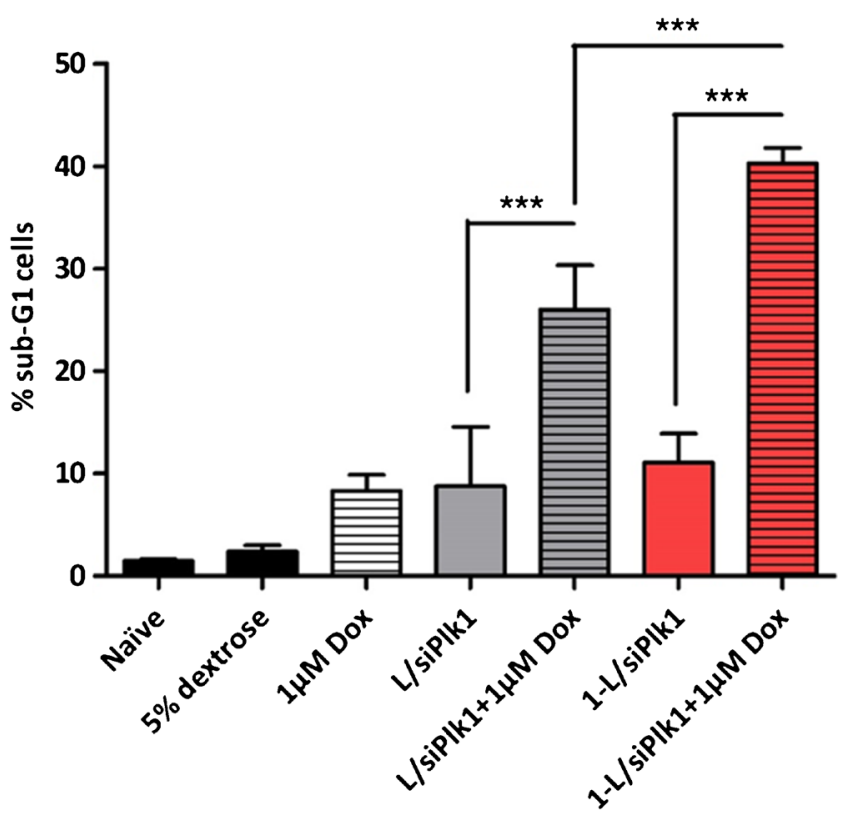

Fig. 8 Growth inhibition and induction of apoptosis in A549 cells by the codelivery of siPLKI and Dox with DOTAP:chol liposomes (L) or ox-MWNT$\mathrm{NH}_{3}{ }^{+}$-DOTAP:chol hybrid (I-L). Sub-GI analysis using propidium iodide staining and flow cytometry was performed $48 \mathrm{~h}$ after transfection. Cells treated with I $\mu \mathrm{M}$ of Dox with or without PLKI silencing with $\mathbf{L}$ or $\mathbf{I}-\mathbf{L}$ vectors. siNEG transfected cells were used as negative controls and the toxicity of each system was normalized to its siNEG control. Results were expressed as mean \pm SD $(n=5)$.

liposome hybrids for the simultaneous delivery of siRNA and an anticancer drug to cancer cells.

We demonstrated that siPLK1 delivery by I-L led to significant silencing of the target protein and that a synergistic effect was achieved when Dox and siPLK1 were co-delivered to A549 cells in vitro. All hybrids displayed $\zeta$-potential values comparable to that of cationic liposomes (Table I) which indicates that $f$-MWNTs incorporation did not affect the overall cationic surface charge of the final hybrids formed and so that they could still be used to complex siRNA. The minimal N/P ratio for full complexation of siRNA and $f$-MWNT-cationic liposome hybrids was found to be 4:1 (Fig. 3), similar to that obtained for cationic liposome and consistent with previous studies.

The fact that the overall charge of liposome and complexation ability was not affected upon hybrid formation suggests that $f$-MWNTs were not associated with the liposome surface, so hydrophobic interactions were proposed. To address this question, NR fluorescence spectroscopy was carried out to investigate the nature of interaction between $f$-MWNTs and cationic liposomes. If the interaction of the $f$-MWNTs is to occur with the lipid bilayer, this should cause a localized disruption in its environment thus altering its microenvironment polarity and therefore changing the spectral characteristics of NR. We hypothesize that upon interaction of liposomes with $f$-MWNTs, the bilayer becomes leakier and hence more polar, leading to a decrease in NR fluorescence intensity. The fact that NR fluorescence was lower for all hybrids, of low $f$ MWNT content, compared to $\mathbf{L}$ could indicate the presence of interaction between the $f$-MWNTs and cationic liposomes. The quenching of dye fluorescence in the presence of CNTs was seen at high $f$-MWNT concentrations agreeing with a previously reported study (27).

Instability poses a major problem in siRNA applications because it degrades in serum within only a few minutes. We tested the ability of the different $f$-MWNT-liposome hybrids to protect siRNA from nuclease mediated degradation by challenging the siRNA complexes with serum and RNAse. Agarose gel electrophoresis results (Fig. 5) indicated that hybrids $\mathbf{I}-\mathbf{L}, \mathbf{I}-\mathbf{H}$, and $\mathbf{2}-\mathbf{L}$ confer similar siRNA protection abilities to cationic liposomes under test conditions. On the other hand, siRNA complexes formed with hybrid $\mathbf{2}-\mathbf{H}$ seem to be less stable, proved by the lower intensity of siRNA bands compared to all other bands. A possible explanation for the lowest stability of hybrid $\mathbf{2}-\mathbf{H}$ and hence the reduced protection ability may be due to the poor dispersibility of $\mathrm{MWNT}-\mathrm{NH}_{3}{ }^{+}$in aqueous solution, potentially leading to particle aggregation and consequent disruption of liposome structure. This result is consistent with NR fluorescence data where $\mathrm{MWNT}-\mathrm{NH}_{3}{ }^{+}$ displayed a lower degree of interaction with cationic liposomes compared to ox- MWNT- $\mathrm{NH}_{3}{ }^{+}$. Taking into account the low stability of hybrid type $\mathbf{2}$ and the fact that lower amounts of CNTs are preferred, next experiments were carried out with hybrid $\mathbf{I}-\mathbf{L}$.

To study the uptake of carriers $\mathbf{L}$ and $\mathbf{I}-\mathbf{L}$ and of siRNA in A549 cells, NR and ATTO 655 labeled-siNEG fluorescence were monitored, respectively. SSC results showed increased cell complexity for I-L compared to $\mathbf{L}$ (Fig. 6a and b), suggesting enhanced cell uptake with $\mathbf{I}-\mathbf{L}$. Furthermore, cells incubated with I-L complexes showed increased NR and ATTO 655 fluorescence compared to $\mathbf{L}$ (Fig. 6c and d). The enhanced siRNA uptake with this hybrid could be due to enhanced cellular internalization (compared to L) (Fig. 6b). Overall, the enhanced cellular uptake of the $f$-MWNT-cationic liposome hybrid compared to cationic liposomes alone revealed a promising relevance of this system for the delivery of genetic material.

Although cell uptake was improved when siRNA was delivered by $\mathbf{I}-\mathbf{L}$ compared to $\mathbf{L}$, both Luciferase and PLK1 silencing experiments revealed comparable gene silencing activity of $\mathbf{I}-\mathbf{L}$ and $\mathbf{L}$ (Fig. 7a and b).

I-L was then utilized as a co-delivery system for the delivery of siRNA and a cytotoxic drug in vitro. The rationale behind combining PLK1 silencing with chemotherapy was that combined therapies may prolong G2/M phase arrest, rendering cells more prone to apoptosis instead of mitotic slippage (28). Sub-G1 analysis was performed in A549 cells to evaluate cellular apoptosis induced by PLK1 depletion and Dox toxicity using $\mathbf{L}$ and $\mathbf{I - L}$. It is possible that Dox interacts with MWNTs via $\Pi-\Pi$ stacking of Dox aromatic chromophore groups and MWN'Ts backbone, as we previously reported (29). 
When $1 \mu \mathrm{M}$ Dox was co-delivered with siPLK1, both carriers achieved a synergistic effect which was significantly more pronounced for I-L compared to $\mathbf{L}$. These results correlate well with the enhanced cellular uptake of this system and put forward hybrid $\mathbf{I}-\mathbf{L}$ as a promising system for the co-delivery of siRNA and a cytotoxic drug and encourage further $f$ MWNT-liposome characterization and in vivo studies.

\section{CONCLUSION}

Post-transcriptional gene silencing by double-stranded siRNA constitutes an attractive approach to cancer therapy. However, in order to become a therapeutic modality, novel delivery systems which allow direct siRNA translocation into the cytoplasm of the target cells together with an efficient gene silencing ability are of the most importance. In the present study, f-MWNTcationic liposome hybrids were formulated and tested for their siRNA binding ability, stability, cellular uptake, in vitro gene silencing efficiency, induction of apoptosis and siPLK1 and Dox co-delivery efficiency. DOTAP:chol liposomes were used as an established model for siRNA delivery and used for comparison. All hybrids investigated here were capable of fully complexing with siRNA at N/P charge ratio of $4: 1$. Formulations where the hydrophobic dye Nile Red was embedded in the lipid bilaver were prepared and interaction between $f$-MWNTs and cationic liposomes was confirmed by fluorescence spectroscopy. Hybrids prepared with the lower concentrations of $f$-MWNTs showed serum and RNAse stabilities similar to those obtained for DOTAP:chol liposomes and therefore the subsequent studies were carried out with these systems. Cellular uptake studies in A549-Luc cells demonstrated the enhanced uptake ability of hybrid I-L. Moreover, gene silencing experiments with siLuc and siPLK1 revealed that $\mathbf{I}-\mathbf{L}$ was able to efficiently silence Luciferase and PLK1 genes to levels comparable to cationic liposomes. In addition, flow cytometry analysis of sub-G1 population showed that these systems were capable of inducing cell apoptosis following PLK1 depletion by siPLK1. When Dox was co-delivered with siPLK1 by these systems, a synergistic effect was achieved with both liposomal system $\mathbf{L}$ and hybrid $\mathbf{I}-\mathbf{L}$, with the latter yielding a significantly higher number of apoptotic cells. The results presented here encourage further testing of $\mathbf{I}-\mathbf{L}$ system as a potential vehicle for the co-delivery of siRNA and cytotoxic drugs. Structural modifications that favor the in vivo use (e.g., the incorporation of a fusogenic lipid such as DOPE or PEGylation of the liposome surface) should be considered.

\section{ACKNOWLEDGMENTS AND DISCLOSURES}

K.T.A.J. acknowledges funding from Biotechnology and Biological Sciences Research Council (BB/J008656/1) and
Worldwide Cancer Research (12-1054). W.T.A.J. and S.P. acknowledge funding from Prostate Cancer UK (Grant CDF-12-002). H.A.F.M.H. acknowledges funding from YCCF. ICN2 acknowledges support from the Severo Ochoa Program (MINECO, Grant SEV-2013-0295). K.T.A.J. and B.B. acknowledge the EU FP7-ITN Marie-Curie network programme RADDEL (290023) and COST Action TD1004 for funding.

Open Access This article is distributed under the terms of the Creative Commons Attribution 4.0 International License (http://creativecommons.org/licenses/by/4.0/), which permits unrestricted use, distribution, and reproduction in any medium, provided you give appropriate credit to the original author(s) and the source, provide a link to the Creative Commons license, and indicate if changes were made.

\section{REFERENCES}

1. Wang J, Lu Z, Wientjes MG, Au JL. Delivery of siRNA therapeutics: barriers and carriers. Am Assoc Pharm Sci J. 2010;12(4):492503.

2. DA Laufer SD, Sczakiel G, Restle T. Selected strategies for the delivery of siRNA in vitro and in viwo. In: Erdmann JB, Volker A, editors. RNA technologies. Berlin Heidelberg: Springer; 2010. p. 29-58.

3. Uddin SN. Cationic lipids used in non-viral gene delivery systems. Biotechnol Mol Biol Rev. 2007;2(3):58-67.

4. Nunes A, Amsharov N, Guo C, Van den Bossche J, Santhosh P, Karachalios TK, et al. Hybrid polymer-grafted multiwalled carbon nanotubes for in vitro gene delivery. Small. 2010;6(20):2281-91.

5. Popov VN. Carbon nanotubes: properties and application. Mater Sci Eng R Rep. 2004;43(3):61-102.

6. Zhang Y, Bai Y, Yan B. Functionalized carbon nanotubes for potential medicinal applications. Drug Discov Today. 2010;15(1112):428-35

7. Moghimi SM, Hunter AC, Murray JC. Nanomedicine: current status and future prospects. J Fed Am Soc Exp Biol. 2005;19(3): 311-30.

8. Kesharwani P, Gajbhiye V, Jain NK. A review of nanocarriers for the delivery of small interfering RNA. Biomaterials. 2012;33(29): 7138-50.

9. Battigelli A, Wang JTW, Russier J, Da Ros T, Kostarelos K, AlJamal KT, et al. Ammonium and guanidinium dendron-carbon nanotubes by amidation and click chemistry and their use for siRNA delivery. Small. 2013;9(21):3610-9.

10. Strebhardt K, Ullrich A. Targeting polo-like kinase 1 for cancer therapy. Nat Rev Cancer. 2006;6(4):321-30.

11. Guerreiro AS, Fattet S, Kulesza DW, Atamer A, Elsing AN, Shalaby T, et al. A sensitized RNA interference screen identifies a novel role for the PI3K pl logamma isoform in medulloblastoma cell proliferation and chemoresistance. Mol Cancer Res. 201 1;9(7): 925-35.

12. Maire V, Nemati F, Richardson M, Vincent-Salomon A, Tesson B, Rigaill G, et al. Polo-like kinase 1: a potential therapeutic option in combination with conventional chemotherapy for the management 
of patients with triple-negative breast cancer. Cancer Res. 2013;73(2):813-23.

13. Nakouzi NA, Cotteret S, Commo F, Gaudin C, Rajpar S, Dessen $\mathrm{P}$, et al. Targeting CDC25C, PLK1 and CHEK1 to overcome docetaxel resistance induced by loss of LZTS1 in prostate cancer. Oncotarget. 2014;5(3):667-78.

14. Sakurai Y, Hatakeyama H, Akita H, Harashima H. Improvement of doxorubicin efficacy using liposomal anti-polo-like kinase 1 siRNA in human renal cell carcinomas. Mol Pharm. 2014;11(8): 2713-9.

15. Georgakilas V, Kordatos K, Prato M, Guldi DM, Holzinger M, Hirsch A. Organic functionalization of carbon nanotubes. J Am Chem Soc. 2002;124(5):760-1.

16. Al-Jamal KT, Gherardini L, Bardi G, Nunes A, Guo C, Bussy C, et al. Functional motor recovery from brain ischemic insult by carbon nanotube-mediated siRNA silencing. Proc Natl Acad Sci U S A. 2011;108(27):10952-7.

17. Al-Jamal KT, Toma FM, Yilmazer A, Ali-Boucetta H, Nunes A, Herrero MA, et al. Enhanced cellular internalization and gene silencing with a series of cationic dendron-multiwalled carbon nanotube:siRNA complexes. J Fed Am Soc Exp Biol. 2010;24(11):4354-65.

18. Ali-Boucetta H, Al-Jamal KT, Muller KH, Li S, Porter AE, Eddaoudi A, et al. Cellular uptake and cytotoxic impact of chemically functionalized and polymer-coated carbon nanotubes. Small. 2011;7(22):3230-8.

19. Ali-Boucetta H, Al-Jamal KT, Kostarelos K. Cytotoxic assessment of carbon nanotube interaction with cell cultures. Methods Mol Biol. 201 1;726:299-312.

20. Samori C, Ali-Boucetta H, Sainz R, Guo C, Toma FM, Fabbro C, et al. Enhanced anticancer activity of multi-walled carbon nanotube-methotrexate conjugates using cleavable linkers. Chem Commun. 2010;46(9):1494-6.

21. Horiba S. Carbon nanotube applications: scientific H; 2014. Available from: http://www.horiba.com/scientific/products/ particle-characterization/applications/carbon-nanotubes/.

22. Lacerda L, Ali-Boucetta H, Kraszewski S, Tarek M, Prato M, Ramseyer C, et al. How do functionalized carbon nanotubes land on, bind to and pierce through model and plasma membranes. Nanoscale. 2013;5(21):10242-50.

23. Xu XY, Xie K, Zhang XQ, Pridgen EM, Park GY, Gui DS, et al. Enhancing tumor cell response to chemotherapy through nanoparticle-mediated codelivery of siRNA and cisplatin prodrug. Proc Natl Acad Sci U S A. 2013;110(46):18638-43.

24. Saraswathy M, Gong SQ. Recent developments in the co-delivery of siRNA and small molecule anticancer drugs for cancer treatment. Mater Today. 2014;17(6):298-306.

25. Karchemski F, Zucker D, Barenholz Y, Regev O. Carbon nanotubes-liposomes conjugate as a platform for drug delivery into cells. J Control Release. 2012;160(2):339-45.

26. Miyako E, Kono K, Yuba E, Hosokawa C, Nagai H, Hagihara Y. Carbon nanotube-liposome supramolecular nanotrains for intelligent molecular-transport systems. Nat Commun. 2012;3:1226.

27. Yang RH, Jin JY, Chen Y, Shao N, Kang HZ, Xiao Z, et al. Carbon nanotube-quenched fluorescent oligonucleotides: probes that fluoresce upon hybridization. J Am Chem Soc. 2008;130(26):8351-8.

28. Degenhardt Y, Lampkin T. Targeting polo-like kinase in cancer therapy. Clin Cancer Res. 2010;16(2):384-9.

29. Ali-Boucetta H, Al-Jamal KT, McCarthy D, Prato M, Bianco A, Kostarelos K. Multiwalled carbon nanotube-doxorubicin supramolecular complexes for cancer therapeutics. Chem Commun. 2008;4:459-61. 